Asymptotic EXPanstons OF INTEgRal Functions OF ZERo ORDER. 209

\title{
ON THE DIRICHLET SERIES AND ASYMPTOTIC EXPANSIONS OF INTEGRAL FUNCTIONS OF ZERO ORDER
}

\author{
By J. E. LitrLeWOod.
}

[Received June 29th, 1908.-Read November 12th, 1908.-Revised Jan.-Feb., 1909.]

\section{Introduction.}

IF an integral function $F(z)$, of finite or zero order, has $-a_{1},-a_{2}, \ldots$ for its sequence of zeros, the Dirichlet series $\sum_{n=1}^{\infty} a_{n}^{-s}$ converges when the real part of $s$ is greater than some finite number $k$, not less than zero, and, for this range of values of $s$, defines an analytic function of $s$. If the sequence $a_{1}, a_{2}, \ldots$ is perfectly general, the line $2 R=k$ is a barrier of essential singularities of the function. If, however, $a_{n}$ is an analytic function of $n$, it will usually happen that the function defined by the Dirichlet series can be continued across the line $2 h=k$, and that it gives rise to an analytic function $S(s)$, whose finite singularities are isolated points. M. Mellin has shown that when this is the case there is an intimate relation between the function $S(s)$ and the asymptotic expansion of the function $\log F(z)$.*

The typical function of finite non-zero order has $-n^{\rho}$ for its $n$-th zero. The Dirichlet series $\Sigma n^{-\rho s}$ then leads to Riemann's function $\xi(\rho s)$, and the properties of this function and its generalisations play an important part in the theory of Dirichlet series and asymptotic expansions of functions of finite order.t

In the integral functions of zero order, we have, for all values of $\rho$, $\left|a_{n}\right|>n^{\rho}$ when $n$ is sufficiently great. There is no single functional form for $a_{n}$ that is typical in the sense in which $n^{\rho}$ is typical in the case of finite non-zero order, but an unlimited number of such forms. Thus, starting from $e^{n}$, we have among possible forms of $a_{n}$,

$$
e^{n^{k}}, \quad \exp \left(e^{n^{\rho}}\right), \quad \exp \exp \left(e^{n^{\rho}}\right), \quad \ldots, \quad(k>1, \rho>0),
$$

* Acta Soc. Sci. Fenn., T. xxix., No. 4 (1900). “ Ein Formel für den Logarithmus transcendenter Funktionen von endlichem Geschlecht." [Reprinted Acta Math., T. xxviri. (1904)].

† E. W. Barnes, Proc. London Math. Soc., Ser. 2, Vol. 3, p. 273.

BER. 2. vOL. 7. NO. 1018. 
in ascending order in $n$, and

$e^{n^{k}}, \quad \exp \left[(\log n)^{1+\rho}\right], \quad \exp \left[\log n(\log \log n)^{\rho}\right], \quad \exp \left[\log n\left(\log _{3} n\right)^{\rho}\right], \quad \ldots$,

in descending order.

$(k<1, \rho>0)$,

The first of these two classes may be disposed of very briefly. M. Fabry* has shown that, if $\Sigma b_{n} z^{c}$ be a power series of unit radius of convergence, and if $\left(c_{n+1}-c_{n}\right) \rightarrow \infty$, the unit circle is a barrier of essential singularities of the function. It follows, on taking $z=e^{-s}$, that the function $\Sigma b_{n} e^{-s c_{n}}$ has the line $2 \Omega=0$ for a barrier. Now, although M. Fabry's result assumes the $c$ 's to be integers, we must expect that in all cases when $\left(c_{n+1}-c_{n}\right) \rightarrow \infty, \Sigma b_{n} e^{-s c_{n}}$, and in particular $\Sigma e^{-s c_{n}}$, has $\mathfrak{a} s=0$ for a barrier, for, as we have said above, the general Dirichlet series has $\mathfrak{l h} s=k$ for a barrier.

It will follow that, if $a_{n}$ be any function of $n$ which increases more rapidly than $e^{\rho n}$ for all values of $\rho$, we must expect the Dirichlet series $\sum a_{n}^{-s}$ to have $s=0$ for a barrier, and the function $S(s)$ to exist only where the Dirichlet series converges. The problem of finding the analytic continuation of the series then does not exist.

In this case, M. Mellin's method for determining an asymptotic expansion for $\log \Pi\left(1+z / a_{n}\right)$ cannot be applied, for it involves the integral $\int S(s) \frac{z^{s} d s}{s \sin \pi s}$, taken along the line $2 a s=-l$, and in the present case this integral does not exist.

It is interesting to notice that the functions of this same class exhibit a certain peculiarity, viz., their asymptotic approximation cannot be effected in terms of the ordinary analytic functions of analysis. I have shown in a former papert that we can find, from arithmetical considerations, closely approximate asymptotic expressions for the functions in question, and that these expressions involve other than elementary analytic functions.

It is clear, then, that for this class of functions there can be no theory analogous to that developed by M. Mellin and Dr. Barnes for the functions of finite order.

It is otherwise with the functions for which $a_{n}$ is of our second class,

- Annales de l'Ecole Normale, Oct., 1896.

$\dagger$ Proc. London Math. Soc., Ser. 2, Vol. 5, p. 361, "On the Asymptotio Approximation to Integral Functions of Zero Order." 
i.e., for which $a_{n}$ is of less order than $e^{n}$, and the present paper is occupied with the problem of determining the functions $S(s)$ which are the continuations of the Dirichlet series with certain forms of $a_{n}$ of this class, and with the problem of applying, on the lines of M. Mellin's method, the knowledge of these functions $S(s)$ to the determination of asymptotic expansions for the corresponding integral functions $\Pi\left(1+z / a_{n}\right)$.

It will be observed that we have included the form $a_{11}=e^{n}$ in neither of our two classes. This form occupies a limiting position between these classes, and, as might perhaps be expected from this fact, the integral function $\Pi\left(1+z / e^{n}\right)$ exhibits a number of exceptional characteristics.

The Dirichlet series $\Sigma e^{-n s}$ is the simplest of all such series, and the function $S(s)$, viz., $e^{-s} /\left(1-e^{-s}\right)$, is expressible in terms of elementary functions. The finite singularities of $S(s)$ are seen to be isolated, but they are infinite in number.

M. Mellin* has given an asymptotic expansion of $\log \Pi\left(1+z / e^{n}\right)$, but the finite term in the exprnsion occurs in the form of an infinite series. ${ }^{+}$ It is also noteworthy that this expansion is not merely asymptotic, but exact, the infinite series in descending powers of $z$ being convergent.

Thus the properties of the function $\Pi\left(1+z / e^{n}\right)$ are quite unlike those of $\Pi\left(1+z / e^{n^{k}}\right)(k>1)$ (which belongs to our first class), and we shall see that they also bear little resemblance to the properties of the function

of our second class.

$$
\Pi\left(1+z / e^{n^{k}}\right)(k<1),
$$

\section{Abstract. $\ddagger$}

The simplest function of our second class is that for which we have $a_{n}=\exp \left(\rho n^{1 / k}\right)$, where $2 a \rho>0(k>1)$. Two generalised forms of this function are considered, corresponding to $a_{n}=\exp \left(\rho n^{1, k}\right) n^{-\lambda}$, and $\exp \left[\rho\left(n^{1 k}+\rho_{1} n^{1 / k_{1}}+\ldots+\rho_{\mu} n^{1 / k_{\mu}}\right)\right]$ respectively, where $\lambda$ and $\rho$ may be complex (subject to the condition $3 a_{\rho}>0$ ), but where $k, k_{1}, \ldots, \rho_{1}, \rho_{2}, \ldots$ are restricted to be real, and where we have $k_{r}>k$. These forms of $a_{n}$

" Loc. cit.

$\dagger$ This series exhibits peculiarities analogous to those mentioned in connexion with our first class of functions. It may be remarked that if the integral function be taken to be $\Pi\left(1+z e^{-\mu \omega}\right)$, where $\omega$ is complex, the series in question no longer remains finite for all values of $z$. Cf. my paper cited above, $\$ 12$.

$\ddagger$ The Abstract was added at the request of the Council. 
give rise to the Dirichlet series,

(I.) $\sum_{n=1}^{\infty} \exp \left(-s n^{1 / k}\right) n^{\lambda s}$, and (II.) $\sum_{n=1}^{\infty} \exp \left[-s\left(n^{1 / k}+\rho_{1} n^{1 / k_{1}}+\ldots+\rho_{\mu} n^{1 / k}\right)\right]$, respectively. (The factor $\rho$ is omitted, as we do not thereby impair the generality of the series.) These series converge when $2 s s>0$, and define analytic functions for this range of values of $s$. Analytic functions $S(s)$ are found, which represent the values, for any value of $s$, obtained by the process of continuation from the Dirichlet series.

For the first case we have

$$
S(s)=k \Gamma(k \lambda s+k) s^{-k \lambda s-k}+P(s, \lambda),
$$

where

$$
P(s, \lambda)=\sum_{n=0}^{\infty} \frac{(-s)^{n}}{\Gamma(n+1)} \xi(-n / k-\lambda s) .
$$

In the second case,

$$
S(s)=\psi(s)-\frac{1}{2}+Q(s),
$$

where $Q(s)$ is an integral function of (apparent) order $k /(k-1)$, with the origin for a simple zern, and where

$\psi(s)=k s^{-k} \sum_{m=0}^{\infty}\left[(-)^{n i} \sum_{\Sigma a_{\nu}=m}\left\{\prod_{\nu=1}^{\mu}\left(\frac{\rho_{\nu}^{a_{\nu}}}{\Gamma\left(\alpha_{\nu}+1\right)}\right) \Gamma\left(\sum_{\nu=1}^{\mu} k a_{\nu} / k_{\nu}+k\right) s^{\left.\sum_{1}^{\mu}(1-k / k) k_{\nu}\right)}\right\}\right]$,

the $\Sigma$ inside the square brackets being taken over all positive or zero integral values $a_{1}, a_{2}, \ldots, a_{\mu}$, such that $a_{1}+a_{2}+\ldots+a_{\mu}=m$.

The method by which the first result is obtained (I., $\S 1$ ) may be sketched as follows. We first suppose $s$ and $\lambda$ real and positive. Following the method of Lindelöf," we take a contour $C$ embracing the positive real axis, and cutting it in the point $\alpha$, where $0<\alpha<1$, and consider the integral

$$
I=\int_{C} \frac{e^{-s x^{1 . k}} x^{\lambda s} d x}{e^{2 \pi x_{i}}-1}
$$

taken over $C$. The singularities of the integrand are at the points $n=1,2,3, \ldots$, and we obtain for the Dirichlet series, $S(s)=I$. The integral $I$ may be divided into the three parts

$$
I_{1}=-\int_{C_{1}} e^{-8 x^{1 \cdot k}} x^{\lambda s} d x, \quad I_{2}=\int_{C_{1}} \frac{e^{-s x^{1 / k}} x^{\lambda s} d x e^{2 \pi x_{\iota}}}{e^{2 \pi x \imath}-1},
$$

and

$$
I_{3}=-\int_{C} \cdot \frac{e^{-8 x^{1 / k}} x^{\lambda s} d x e^{-2 \pi x t}}{e^{-2 \pi x t}-1}
$$

* Le Calcul des Résidus, p. 56. 
where $C_{1}$ and $C_{2}$ denote the parts of $C$ respectively above and below the real axis. In $I_{1}$ the contour may be deformed into the line from a to infinity, taken along the real axis, and in $I_{2}$ and $I_{3}, C_{1}$ and $C_{2}$ may be deformed into the streight lines $a+1 \infty$ to $a$, and $a$ to $a-1 \infty$ respectively.

It is easily shown that $I_{1}$ tends to the limit $s^{-k \lambda s-k} k \Gamma(k \lambda s+k)$ as a tends to zero (when $s$ and $\lambda$ are positive), while $I_{2}+I_{3}$ is shown to tend to the limit

$$
22 \pi \int_{0}^{\infty} \exp \left[-s t^{1 / k} e^{\mathrm{j} \pi \iota / l i}\right] t^{\lambda s} e^{\frac{h \pi \tau \lambda s}{\pi}} \frac{\iota d t}{e^{2 \pi t}-1} .
$$

If the factor $\exp \left[-s t^{1 / k} e^{\text {jralk }}\right]$ under the integral sign in this last expression be replaced by its expansion in powers of $s$, and if the integration be effected term by term, we obtain an expression for $I_{2}+I_{3}$ which leads to the result (1). Now the right-hand side of (1) defines an analytic function of the two variables $s$ and $\lambda$ for all values of $s$ and $\lambda$, and we have proved that it represents the Dirichlet series when $s$ and $\lambda$ are real and positive. It follows that it is the analytic function representing all values obtained by continuation, for the variables $s$ and $\lambda$, from the Dirichlet series.

The analytic function (1) is in general multiform, the multiformity depending on $s^{-k \lambda s-k}$. If a cut be made along the negative real axis preventing the point $s$ from making a circuit of the origin, the function obtained by continuation in the cut plane from the Dirichlet series is evidently uniform. [ $\lambda$ is now supposed to be a constant.] It is shown that this function, which is denoted by $S_{0}(s)$ corresponds to the determination

$$
s^{-k \lambda s-k}=\exp [-(k \lambda s+k) \log s],
$$

where $\log s$ has its principal value.

The function $S(s)$ (in the uncut plane) has $s=0$ for its sole finite singularity. The apparent poles at the poles of $\Gamma(k \lambda s+k)$ are neutralised by the poles of $\xi(-n / k-\lambda s)[n=1,2, \ldots]$.

In the particular case when $\lambda$ is zero, the function $P(s, 0)$ becomes an integral function of $s$, whose apparent order is shown to be $k /(k-1)$ (I., $\S 4)$.

The result (2) is established on the sàme general lines (I., § 5). The series for $\psi(s)$ converges for all values of $s$, and $\psi(s)$, and therefore also $S(s)$, evidently has $s=0$ for its sole finite singularity.

The function obtained by continuing the Dirichlet series over the $s$ plane cut along the negative real axis is determined by assigning to every term $s^{\theta}$ of $\psi(s)$ the value exp $(\theta \log s)$, where $\log s$ has its principal value. 
It is important to know something of the behaviour of $\psi(s)$ when $|s|$ is large. It is shown (II., $\$ 4$ ) that, when $|s|$ is sufficiently large, we have

$$
|\psi(s)|<K \exp \left[|s|^{1+\bullet}\right] \text {. }
$$

In order to establish this result it is previously shown (I., $\S 5$ ), that if the series for $\psi(s)$ be arranged in order of non-decreasing indices of $s$, each term of the old expression being retained as a separate term of the new one, ${ }^{*}$ we have

$$
\psi(s)=s^{-k}\left[\Gamma(k+1)+\sum_{n=1}^{\infty} c_{n} s^{\theta_{n}}\right],
$$

where $0<\theta_{1} \leqslant \theta_{2} \leqslant \ldots, \quad \theta_{n}>n^{1(\mu+1)} / K$,

and

$$
\left|c_{n}\right|<\left[K / \theta_{n}\right]^{\theta_{n}} \text {. }
$$

In the case when $a_{n}=\exp \left(n^{1 k}+\rho_{1} n^{1 / k}\right)$, the general formula becomes fairly simple. We have (I., §6)

$$
S(s)=\psi(s)-\frac{1}{2}+Q(s),
$$

where $\quad \psi(s)=s^{-k}\left[\Gamma(k+1)+k \sum_{n=1}^{\infty} \frac{\left(-\rho_{1}\right)^{n} s^{n\left(1-k k_{1}\right)}}{\Gamma(n+1)} \Gamma\left(n k / k_{1}+k\right)\right]$,

so that the series within square brackets is an integral function of the argument $s^{\left(k_{1}-k\right) k_{1}}$ of order $k_{1} /\left(k_{1}-k\right)$.

[It is not important to know the coefficients in the function $Q(s)$. Those of the singular part $\psi(s)$ of $S(s)$, however, appear in the asymptotic expansion of $\log \Pi\left(1+z / a_{n}\right)$.]

Having this knowledge of the functions generated by the Dirichlet series I. and II., we may attack the problem of finding asymptotic expansions for the corresponding integral functions $\Pi\left(1+z / a_{n}\right)$. We are met, however, at the outset, by a difficulty which does not occur in the corresponding theory of the integral functions of finite non-zero order. We require to use the integral $\int \frac{z^{s} S(s) d s}{s \sin \pi s}$, taken along parts of the line 2a $s=-l(l>0)$. This integral will not exist in any case unless $|S(s)|<K \exp (\pi|s|)$. Now the Dirichlet series itself can give us no information as to the behavour of $S(s)$ on $3 s=-l$, for it does not con-

* I.c., if two terms of the old expression have the same index for $s$, they are not to be combined into one term in the new series. 
verge there. On the other hand, if we appeal, for example in our second case, to the formula (2), we only know concerning $Q(s)$ that it is an integral function of order $k /(k-1)$, and therefore that when $|s|$ is large, $|S(s)|<K \exp \left[|s|^{k /(k-1)+e}\right]$. This result is clearly inadequate for our purposes, and it is necessary to provide some means of establishing the better inequality for $|S(s)|$ which in fact exists, for points on the line IR $s=-l$. This means is afforded by the following general theorem, which is proved in II., $\S 1$.

Let $F(z)$ be an analytic function with a finite number of singularities and possessing the following properties :-

(1) In the region $R$ consisting of the whole of the plane on one side of a straight line $L$, and including the points of $L, a$ branch $F_{0}(z)$ of $F(z)$ has no singularities, and for all points of $R$, and for an arbitrarily small $\epsilon$,

$$
\left|F_{0}(z)\right|<K \exp \left(r^{p-1+\varepsilon}\right)
$$

where $\rho>1$, and $r=|z|$.

(2) In the whole plane, assuming the finite singularities to be excluded by small circles of radius $\delta$,

for an arbitrary $\epsilon$.

$$
|F(z)|<K \exp \left(p^{p+e}\right),
$$

Let $L^{\prime}$ be the straight line parallel to $L$ and at a distance $l$ from it on the side of $L$ remote from $R$, and let $R^{\prime}$ be the region of the plane on the same side of $L^{\prime}$ as $R$. Let a system of cuts be made so as to prevent the point $z$, when it is restricted to the region $R^{\prime}$, from circumscribing any singularity of $F(z)$, so that $F_{0}(z)$, when continued over $R^{\prime}$, generates a uniform function. Then, for any arbitrary $\epsilon$, we shall have, for all points of $R^{\prime}$,

$$
\left|F_{0}(z)\right|<K \exp \left(p^{p-1+e}\right) \text {. }
$$

From this theorem it is easily deduced that on $a s=-l$, we have

$$
\left|S_{0}(s)\right|<K \exp \left[-|s|^{1 /(k-1)+e}\right],
$$

where $S_{0}(s)$ denotes the function obtained from the Dirichlet series I. or II. by continuation over the s-plane cut along the negative real axis. Provided, then, that we assume $k>2$, the integral $\int \frac{S_{0}(s) z^{s} d s}{s \sin \pi s}$, takeu over the part of the line ars $=-l$ either above or below the real axis, will exist.*

\footnotetext{
* It should be noted that $s$ cannot describe the whole line continuously in the cut plane.
} 
The problem of finding an asymptotic expansion for $\log F(z)$ is greatly simplified if $S(s)$ is known to be a uniform function. This can never be the case for the function II., but occurs in the case of I., provided $\lambda$ is zero and $k$ is an integer. This simplest case is considered first, and may be treated on the lines of M. Mellin's paper cited above. (II., $\$ \S 2,3$.)

We find that, if

$$
F(z)=\prod_{n=1}^{\infty}\left[1+z / \exp \left(\rho n^{1 / 2}\right)\right],
$$

where $\mathrm{R} \rho \mathbf{0}$, and where $k$ is an integer not less than $\mathbf{3}$,

$$
\begin{aligned}
\log F(z)=(k+1)^{-1} & \rho^{-k}(\log z)^{k+1} \\
& \left.+2(k+1)^{-1} \rho^{-k} \sum_{n=1}^{k}{ }_{k+1} C_{2 n}\left(2^{2 n-1}-1\right) \pi^{2 n} B_{2 n} \log z\right)^{k+1-2 n} \\
& -\frac{1}{2} \log z-\rho \xi(-1 / k)+\sum_{m=1}^{N}(-)^{m-1} \frac{S(-\rho m)}{m} z^{-m}+J_{N},(6)
\end{aligned}
$$

where $k=\frac{1}{2} k$ or $\frac{1}{2}(k+1)$, according as $k$ is even or odd, the B's are Bernoulli's numbers, $S(s)$ is the function (1) (with $\lambda=0$ ), and where

$$
\lim _{1: i \rightarrow \infty}\left|J_{N} z^{N}\right|=0 \text {. }
$$

This expansion clearly cannot be valid in the vicinity of the zeros of $F(z)$. It is, in fact, established first for the case when $z$ is excluded from the spiral strip contained by the curves $z=-\exp \left(\rho x \pm \delta_{\iota}\right)$, where $\delta$ is an arbitrarily small positive number, and where $x$ takes all real values from $-\infty$ to $+\infty(\S 2)$. The region thus excluded is of the type usually considered in the theory of asymptotic expansions. It is then shown (\$ 3$)$ that the region from which $z$ is excluded may be narrowed (outside the circle $|z|=1$ ) into the spiral strip between the curves

$$
z=\exp \left[\rho x \pm i \delta(\log r)^{-(k-2)+\delta}\right]
$$

where $\delta$ is arbitrarily small.

If $\lambda$ is not zero, the form of the singularity at $s=0$ of the function defined by I. makes the extension of our methods to this case, if not impossible, at any rate a matter of great difficulty.

For the function $S(s)$ defined by II., however, the singular part $\psi(s)$ is the sum of an infinite number of terms of the form $c_{n} s^{\theta_{n}}$, and, although the sum represents a complicated singularity, we are able to consider the effect of each term separately. We consider the function

$$
F(z)=\prod_{n=1}^{\infty}\left(1+z / a_{n}\right),
$$


1908.] AsYmptotic eXpansions OF INTEGRaL FUnCtions OF ZERo ORDER. 217

where

$$
a_{n}=\exp \left[\rho\left\{n^{1 / k}+\rho_{1} n^{1 / k_{1}}+\rho_{2} n^{1 / k_{2}}+\ldots+\rho_{\mu} n^{1 / k_{\mu}}\right\}\right],
$$

and where $k_{\mu}>\ldots>k_{1}>k>2$, and $\mathfrak{a} \rho>0$.

The assumption $k>2$ is certainly necessary in order that we may have $\left|S_{0}(s)\right|<\exp (\epsilon|s|)$ on ala $s=-l$, and it seems necessary to this end to introduce the additional restriction that $\rho_{1}, \rho_{2}, \ldots$ shall be real. With these assumptions the inequality for $S_{0}(s)$ is readily deduced from the general theorem given above.

The following expansion is obtained (II., $\S \S 4,5$ )

$\log F(z)=-\rho\left[\xi(-1 / k)+\sum_{\nu=1}^{\mu} \rho_{\nu} \xi\left(-1 / k_{\nu}\right)\right]-\frac{1}{2} \log z$

$$
+\sum_{n=0} \frac{\rho c_{n} \sin \left(k-\theta_{n}\right) \pi}{\pi}\left(\frac{\log z}{\rho}\right)^{k-\theta_{n}+1} \sigma_{n}
$$

where

$\sigma_{n}=\Gamma\left(\theta_{n}-k-1\right)+2 \sum_{m=1} \Gamma\left(2 m+\theta_{n}-k-1\right) \frac{\left(2^{2 i n-1}-1\right) \pi^{2 i n}}{2 m !} B_{2 m}(\log z)^{-2 m}$

where $s^{-k} \sum_{n=0}^{\infty} c_{n} s^{\theta_{n}}$ is the series (5). The infinite series is to be arranged in order of increasing indices of $1 / \log z$, and, when we stop at aniy term, say of order $(\log z)^{-p}$, we have, if $R$ is the remainder,

$$
\lim _{|z| \rightarrow \infty}\left|R(\log z)^{p}\right|=0 .
$$

In the proof it is assumed for simplicity that $\rho$ is real, and that $z$ is excluded from the strip between the straight lines

$$
z=\exp \left[\rho\left\{x^{1 / k}+\rho_{1} x^{1 / k_{1}}+\ldots+\rho_{\mu} x^{1 k_{\mu}}\right\} \pm \iota \delta\right] ;
$$

the expansion may, however, be proved for $\rho$ complex, and when $z$ is excluded from a narrower spiral strip.

We have, by the ordinary theory of residues,

$$
\log F(z)=-\frac{1}{2 \iota} \int_{C} \frac{z^{*} S_{0}(\rho s) d s}{s \sin \pi s},
$$

where $S_{0}(\rho s)$ is the function derived by continuation, in the plane cut along the negative real axis, from the Dirichlet series II. (with $\rho s$ as argument instead of $s$ ), and where the contour $C$ embraces the positive real axis and passes between the points 0 and 1 . The contour $C$ may be deformed into the contour $C^{\prime}$ consisting of the line from $-l+\infty$ l to $-l$ $(0<l<1)$, the circle $|z|=l$ described clockwise from $-l$ to $-l$, and 
the line from $-l$ to $-l-\infty$. Each term of $S_{0}(\rho s)$ of the form $c_{n}(\rho s)^{\theta_{n}-k}$ is then shown to contribute a divergent but asymptotic series proceeding in descending (non-integral) powers of $\log z$; while the uniform part $-\frac{1}{2}+Q(\rho s)$ contributes the first two terms on the right-hand side of (7). The necessity of dealing with an infinite series of terms, each of which generates a divergent series, is the main cause of the complexity of the proof of the final expansion.

The line of proof adopted is difficult to extend to the case when $\rho_{1}, \rho_{2}, \ldots$ are complex, and it must break down when $k<2$. The expan. sion, however, is valid in each case, and even when $k_{1}, k_{2}, \ldots$ (but not $k$ ) are complex, provided that $a 1 / k_{\nu}<1 / k<1$. The proof of this, however, cannot be given here. It depends on a comparison of the known form of the expansion in our restricted case with a general form obtained by an entirely different method, which is mainly arithmetical in character. This method is itself unable to determine the general coefficients of its result, but it is easily shown that they must be those occurring in (7).*

There is, however, one interesting point which this principle is unable to decide, as to whether the expansion (6) for the case $a_{n}=\exp \left(\rho n^{1, k}\right)$ (where $k$ is an integer), which contains an asymptotic series in descending powers of $z$, is valid when $k=2$.

It is not possible to apply pur methods to determine asymptotic expansions for $\log F(z)$ in cases where $\left|a_{n}\right|$ is of less order in $n$ than exp $\left(n v^{2}\right)$ for arbitrarily small values of $\epsilon$. (On this point, see II., $\S$ 8.) The functions $S(s)$ generated by the corresponding Dirichlet series $\Sigma a_{n}^{-s}$ can, however, be determined in a number of cases. In Part I. (\$\$ 7-9) the following series are considered :-

$$
\text { (III.) } \sum_{n=2}^{\infty} \exp \left[-s(\log n)^{1+k}\right], \quad k>0 \text {; }
$$

and its generalisation

$$
\Sigma \exp \left[-s\left\{(\log n)^{1+k}+\rho_{1}(\log n)^{1+k_{1}}+\ldots+\rho_{\mu}(\log n)^{1+k_{\mu}}\right\}\right],
$$

where $k>k_{1}>\ldots$, and $k>0$;

$$
\text { (IV.) } \quad \sum_{n=3}^{\infty} \exp [-s \log n \log \log n] \text {; }
$$

- I hope shortly to publish an account of this general theory, which applies to all integral functions of our second class which arise in a natural manner, under the title "On a Class of Integral Functions." 
1908:] AsYMptotic eXPANSIONS OF INTEgRAL FUNCTIONS OF ZERO ORDER. 219

and

$$
\sum \exp \left[-s \log n\left(\log _{p} n\right)^{k}\right]
$$

where

$$
\log _{p} n=[\log \log \ldots(p \text { times })] n .
$$

For the series (III.), we find (I., § 7)

$$
S(s)=\psi(s)-\frac{1}{2}+P(s),
$$

where $P(s)$ is an integral function of order unity, and where

$$
\psi(s)=(1+k)^{-1} \sum_{n=0}^{\infty} s^{-(n+1):(1+k)} \frac{\Gamma\{(n+1) /(1+k)\}}{\Gamma(n+1)},
$$

so that $\psi(s)$ is an integral function, of the argument $s^{-1 /(1+k)}$, of order $(k+1) / k$.

For (IV.), we find (I., § 9),

$$
S(s)=s^{-1} T\left(\log s+s^{-1}\right)+P(s),
$$

where $P(s)$ is an integral function of order unity, and $T(x)$ is an integral function of $x$ of infinite order.

In all the cases considered, $S(s)$ is shown to have $s=0$ for its sole finite singularity.

\section{Part I.}

Dirichlet Series.

1. We begin with the series

$$
\sum_{n=1}^{\infty} \exp \left(-s n^{1 / k}\right) n^{\lambda s} \quad(k>1),
$$

where $\lambda$ is any complex number, and $n^{\lambda s}$ is interpreted to mean $\exp (\lambda s \log n)$, $\log n$ having its real value.

We shall establish the following results :-

The function $S(s)$ is

$$
k \Gamma(k \lambda s+k) s^{-k \lambda s-k}+P(s, \lambda),
$$

where

$$
P(s, \lambda)=\sum_{n=0}^{\infty} \frac{(-s)^{n}}{\Gamma(n+1)} \xi(-n / k-\lambda s) .
$$

$S(s)$ has $s=0$ for its sole finite singularity.

If a cut be made in the s-plane along the negative real axis, and if $S_{0}(s)$ be the function which is defined by continuation in the cut plane 
from the Dirichlet series,

$$
S_{0}(s)=k \Gamma(k \lambda s+k) \exp [-(k \lambda s+k) \log s]+P(s, \lambda),
$$

where log s has its principal value.

Let $x^{1 / k}$ be interpreted, as usual, to mean $\exp (\log x / k)$, where $\log x$ is real when $x$ is real and positive.

We shall suppose for the present that $s$ and $\lambda$ are real and positive. Consider the integral

$$
I=\int_{L_{1}+L_{2}} e^{-s x^{1: k}} \frac{x^{\lambda s} d x}{e^{2 \pi x_{i}}-1}
$$

taken along the contour $L_{1}+L_{2}$, where $L_{1}$ consists of the straight lines $+\infty+\imath \beta$ to $\alpha+\imath \beta$ to $\alpha ; L_{2}$ of the lines $\alpha$ to $\alpha-\imath \beta$ to $+\infty-\imath \beta, \beta$ being any positive number, and $\alpha$ being any number between 0 and 1 .

The integrand is a uniform function of $x$ for $\mathfrak{R} x>0$, and the integral is convergent at infinity. Also the sum of the residues of the integrand at those singularities which lie inside $L_{1}+L_{2}$, viz., the points $1,2,3, \ldots$, is $\frac{1}{2 \pi \iota} \sum_{n=1}^{\infty} e^{-s n^{1 . k}} n^{\lambda s}$, which series is convergent and has $\frac{1}{2 \pi \iota} S(s)$ for its sum. Thus we have

$$
I=S(s) .
$$

Now we have

$$
I=-\int_{L_{1}} e^{-s x^{1 k}} x^{\lambda s} d x+\int_{L_{1}} e^{-s x^{1, k}} \frac{x^{\lambda s} d x}{1-e^{-2 \pi x}}+\int_{L_{2}} e^{-s x^{1 k}} \frac{x^{\lambda s} d x}{e^{2 \pi x}-1} .
$$

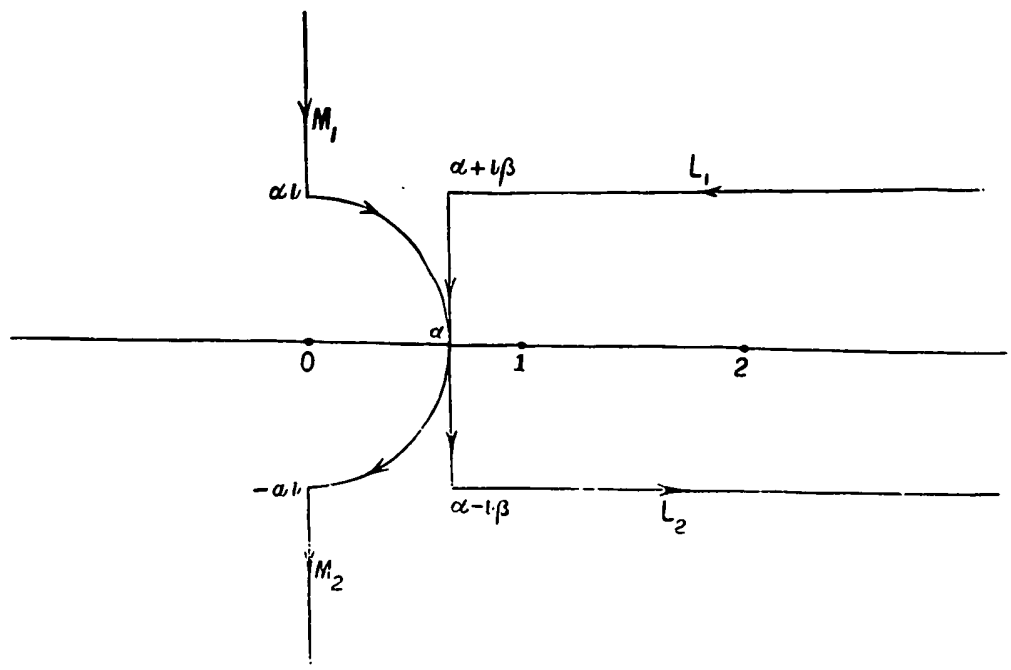


Let $M_{1}$ be the straight line from $+\infty$ l to al, together with the quadrant of the circle $|x|=\alpha$ from $\alpha$ to $\alpha$, and let $M_{2}$ be the reflection of $M_{1}$ in the real axis.

Now $\int e^{-s x^{1 / k}} \frac{x^{\lambda s} d x}{1-e^{ \pm 2 \pi x_{1}}}$, taken along a part of the circle $|x|=R$, intercepted either between $L_{1}$ and $M_{1}$, or between $M_{2}$ and $L_{2}$, tends to zero as $R$ tends to infinity. For, if $x$ be any point of either piece of the circle, we have

$$
\lim _{k \rightarrow \infty}\left|x^{2} \cdot e^{-s 1^{1 / k}} x^{\lambda s} \frac{1}{1-e^{ \pm 2 \pi x}}\right|=0 .
$$

Hence, in the last two integrals on the right-hand side of (2), we may deform $L_{1}$ into $M_{1}$, and $L_{2}$ into $M_{2}$, since we do not thereby pass over singularities of the integrands. We therefore have, from (1) and (2),

where

$$
\left.\begin{array}{rl}
S(s) & =I_{1}+I_{2}+I_{3} \\
I_{1} & =-\int_{L_{1}} e^{-s x^{1, k}} x^{\lambda s} d x \\
I_{2} & =-\int_{M_{1}, \frac{3}{3} \pi} e^{-s x^{1 ; k}} x^{\lambda s} \frac{d x}{e^{-2 \pi x}-1} \\
I_{3} & =\int_{M_{2}, 0} e^{-s x^{1 / k}} x^{\lambda s} \frac{d x}{e^{2 \pi x i}-1}
\end{array}\right\},
$$

where $\int_{C, \phi}$ means that the integral is taken along the contour $C$, and that $x$ is supposed to have the argument $\phi$ at the starting point of $C$.

First consider the integral $I_{1}$.

The contour $L_{1}$ may be deformed into the straight line from $+\infty$ to $a$ along the real axis, since the integral is convergent at infinity, and since we do not pass over singularities of the integrand. Hence

$$
\begin{aligned}
I_{1} & =-\int_{\infty}^{a} e^{-s x^{1 \cdot k}} x^{\lambda s} d x \\
& =s^{-k \lambda s-k} \int_{(\alpha / s)^{k}}^{\infty} e^{-y} y^{k \lambda s} k y^{k-1} d y,
\end{aligned}
$$

when we write

$$
x=(y / s)^{k} .
$$

Since $\lambda s>0$, this last integral is convergent at the origin. Hence, 
making $a$ tend to zero, we have

$$
\begin{aligned}
I_{1} & =k s^{-k \lambda s-k} \int_{0}^{\infty} e^{-y} y^{k \lambda s+k-1} d y+\epsilon(\alpha) \\
& =s^{-k \lambda s-k} k \Gamma(k \lambda s+k)+\epsilon(\alpha) .^{*}
\end{aligned}
$$

Next consider $I_{2}$ and $I_{9}$.

On the straight portion of $M_{1}$, since $x$ starts at $+\infty$, with the argument $\frac{1}{2} \pi$, we have $x=t e^{\frac{k \pi}{m}}$ and $x^{1 k}=t^{1 k} e^{\frac{\lambda \pi}{2} k}$, where $t$ is real, and similarly, on the straight portion of $M_{2}, x^{1 k}=t^{1 / k} e^{-\frac{1}{k} \pi c^{k}}$. Hence, dividing $I_{2}$ and $I_{3}$ into parts corresponding to the straight and curved parts of the respective contours, we have

$$
\begin{aligned}
& I_{2}=I_{2}^{\prime}+I_{2}^{\prime \prime} \\
& I_{3}=I_{3}^{\prime}+I_{3}^{\prime \prime} \\
& I_{2}^{\prime}=-\int_{\infty}^{a} \exp \left[-s t^{1 k} e^{\frac{3 \pi t / l}{2}}\right] t^{\lambda s} e^{\frac{\eta \pi}{\pi} \lambda s} \frac{\iota d t}{e^{2 \pi t}-1}
\end{aligned}
$$

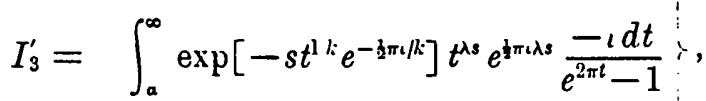

$$
\begin{aligned}
& I_{: \prime}^{\prime \prime}=\int_{a 1,3 \pi}^{a} e^{-s x^{\prime k}} x^{\lambda s} \frac{-d x}{e^{-2 \pi x i}-1} \\
& I_{s}^{\prime \prime}=\int_{a, 0}^{-a,} e^{-s x^{\prime k}} x^{\lambda s} \frac{d x}{e^{2 \pi x_{1}}-1}
\end{aligned}
$$

where

the last two integrals being taken along arcs of the circle $|x|=a$.

It may be shown that $\quad I_{2}^{\prime \prime}+I_{s}^{\prime \prime}=\epsilon(a)$.

If $x$ be a point of the contour of $I_{2}^{\prime \prime}$ or of $I_{3}^{\prime \prime}$, we have

$$
\left|e^{-s 1^{1 / k}} x^{\lambda s} \frac{1}{e^{ \pm 2 \pi x i}-1}\right|<K|x|^{\lambda s-1}
$$

where $K$ does not depend on $a$. It follows, since $\lambda s>0$, that $I_{2}^{\prime \prime}$ and $I_{3}^{\prime \prime}$ tend to zero with $a$, and we have the result (6).

Returning to (5), let us now consider $I_{2}^{\prime}+I_{3}^{\prime}$.

- I shall always use $\mathrm{\epsilon}(x)$ to mean a function of $x$ which tends to zero as $x$ tends to its limit, and shall use the same symbol for all such functions. In the same way to express the fact that $|f(x)|$ is always less than some finite number, I shall write $|f(x)|<K$, and shall always use the same $K$. 


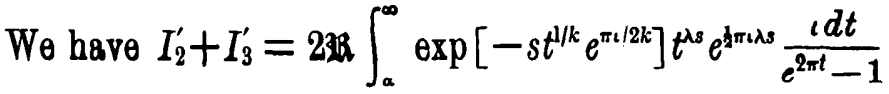

$$
\begin{aligned}
& =2 \mathfrak{A} \int_{0}^{\infty} \exp \left[-s t^{1 / k} e^{\pi t / 2 k}\right] t^{\lambda s} e^{\frac{\lambda}{2 \pi \iota \lambda s}} \frac{\iota d t}{e^{2 \pi t}-1}+\epsilon(a),
\end{aligned}
$$

since the last integral is convergent at $t=0$, on account of the factor $t^{\lambda s}$. Calling the integral on the right-hand side of (7) $I_{4}$, we have, from (3), (4), (5), (6), (7),

$$
S(s)=k \Gamma(k \lambda s+k) s^{-k \lambda s-k}+I_{4}+\epsilon(\alpha) ;
$$

and therefore, since every term but the lest is independent of $a$,

$$
S(s)=k \Gamma(k \lambda s+k) s^{-k \lambda s-k}+I_{4} .
$$

We shall now study the integral $I_{4}$, where $s$ and $\lambda$ are still supposed real and positive.

We have $\quad \exp \left[-s t^{1 / k} e^{\pi / 2 k}\right]=\sum_{n=0}^{\infty} \frac{(-s)^{n}}{\Gamma(n+1)} e^{n \pi \imath 2 k} t^{n / k}$.

Multiplying by $e^{\pi \cdot \lambda t} \cdot 2 t t^{\lambda s} /\left(e^{2 \pi t}-1\right)$, and integrating term by term from $t=0$ to infinity, ${ }^{*}$ we have

$$
\begin{aligned}
I_{4} & =\mathbb{R} 2 t e^{\frac{1 \pi \tau}{2 \pi \lambda}} \sum_{n=0}^{\infty} \frac{(-s)^{n}}{\Gamma(n+1)} e^{\frac{1}{2 n \pi / k}} \int_{0}^{\infty} \frac{t^{n / k+\lambda s}}{e^{2 \pi t}-1} d t \\
& =\sum_{n=0}^{\infty} \frac{(-s)^{n}}{\Gamma(n+1)}\left[-2 \sin \left\{\frac{1}{2} \pi(n / k+\lambda s)\right\}\right] \int_{0}^{\infty} \frac{t^{n^{\prime} k+\lambda s}}{e^{2 \pi t}-1} d t .
\end{aligned}
$$

Now when $\mathfrak{a} z>0$, we have

$$
\int_{0}^{\infty} \frac{t^{z} d t}{e^{2 \pi t}-1}=-\frac{1}{2} \operatorname{cosec}\left(\frac{1}{2} \pi z\right) \dot{\zeta}(-z)
$$

We therefore have

$$
I_{4}=\sum_{n=0}^{\infty} \frac{(-s)^{n}}{\Gamma(n+1)} \xi(-n / k-\lambda s)
$$

We now obtain from (8) the result

$$
S(s)=k \Gamma(k \lambda s+k) s^{-k \lambda s-k}+\sum_{n=0}^{\infty} \frac{(-s)^{n}}{\Gamma(n+1)} \xi(-n / k-\lambda s) .
$$

- The justifioation of this step offers no special difficulty, either here or elsewhere where it occurs in the paper. I shall omit the proofs throughout. 
Using the formula

$$
\xi(z)=2(2 \pi)^{z-1} \sin \left(\frac{1}{2} \pi z\right) \Gamma(1-z) \xi(1-z),
$$

we obtain from (9) the alternative form

$$
\begin{aligned}
& S(s)=k \Gamma(k \lambda s+k) s^{-k \lambda s-k} \\
&+2(2 \pi)^{-\lambda s-1} \sum_{n=0}^{\infty}\left[\frac{(-)^{n-1} s^{n}}{\Gamma(n+1)} \sin \left\{\frac{1}{2} \pi(n / k+\lambda s)\right\}\right. \\
&\times \Gamma(n / k+\lambda s+1) \xi(n / k+\lambda s+1)] .
\end{aligned}
$$

The results (9) and (9)' have been established on the assumption that $s$ and $\lambda$ are real and positive. But if $s$ and $\lambda$ are any complex num. bers such that $-(n+k \lambda s) / k$ is not unity [i.e., a singularity of $\Gamma(1-z)$ ] for any value of $n$ from 0 to infinity, the series on the right-hand side of $(9)^{\prime}$ is convergent.*

Also it can be shown that the right-hand side of $(9)^{\prime}$ can be differentiated term by term with respect to $s$ or $\lambda$, so that this expression is an analytic function of $s$ and $\lambda$ for all values of $s$ and $\lambda$, except $s=0$ and such pairs of values of $s$ and $\lambda$ as make $k(1-\lambda s)$ zero or a positive integer.

Now the Dirichlet series defines an analytic function of $s$ and $\lambda$ for the range of values defined by $\mathfrak{k} s>0, \lambda$ being allowed to take any value. Moreover, we have seen that when $s$ and $\lambda$ are real and positive, the right-hand side of (9) or of (9)' agrees in value with the Dirichlet series. It follows that the right-hand side of (9) is the analytic function of $s$ and $\lambda$ obtained by continuation from the Dirichlet series; or, since $\lambda$ is supposed to have a constant value, we may say that for any complex value of $\lambda$, the right-hand side of (9) defines, for all values of $s$ except isolated singularities, the analytic function of $s$ obtained by continuation from the Dirichlet series, which is detined for ans $>0$. This is the first of our results.

- For $\sin \left\{\frac{1}{2} \pi(n / k+\lambda s)\right\}$ and $\zeta(n / k+\lambda s+1)$ are finite for all values of $n$, while when $n$ is large $|\Gamma(n / k+\lambda s+1) / \Gamma(n+1)|$ behaves like $n^{-[(k-1) / k] n}$. The series therefore converges like $\Sigma[s / ; 2 \pi n k-1), k\}]^{n}$. 
2. We shall now establish the third result.

The function $P(s, \lambda)$ is uniform, so also is $\Gamma(k \lambda s+k): s^{-k \lambda s-k}$ means $\exp [-(k \lambda s+k) \log s]$, and the question we have to decide is the relation between the determinations of $\log s$ and the various branches of $S(s)$ as arising from the primary branch defined by the Dirichlet series for 2hs $>0$.

The term $n^{\lambda s}$ which occurs in the Dirichlet series meens exp $(\lambda s \log n)$, where $\log n$ is real and is a uniform function of $\lambda$. Then, for a given real and positive $s$, the Dirichlet series defines a uniform function of $\lambda$. Again, $\log s$ being taken real, the right-hand side of (9) defines a uniform function of $\lambda$. These two uniform functions agree in value for all real and positive values of $\lambda$; they therefore agree for all values of $\lambda$. We may restate this result by saying that for any complex $\lambda$, if $s$ is real and positive, the Dirichlet series is equal to the right-hand side of (9), $\log s$ being taken real.

If now a cut be made in the $s$-plane along the negative real axis, and if $S_{0}(s)$ be the function defined in the cut plane by continuation from the Dirichlet series, then $S_{0}(s)$ must be the continuation in the cut plane of the right-hand side of (9). Now in the cut plane $\log s$, which is real when $s$ is real and positive, must have its principal value, i.e., it must have the modulus of its imaginary part not greater than $\pi$. This gives the desired determination for $s^{-k \lambda s-k}$ corresponding to the branch $S_{0}(s)$.

3. The point $s=0$ is clearly a singularity of $S(s)$. We have to show that $S(s)$ has no other finite singularity.

The possible finite singularities other than $s=0$ are those values of $s$ which make $-(n / k+\lambda s)$ equal to 1 for some positive or zero integral value of $n$. Suppose then that

$$
-\left(n / k+\lambda s_{n}\right)=1:
$$

the term $\xi(-n / k-\lambda s)$ then becomes infinite at $s=s_{n}$.

In the vicinity of this point we have

$$
\xi(-n / k-\lambda s)=\xi\left\{\left(\lambda\left(s_{n}-s\right)+1\right\}=\frac{1}{\lambda\left(s_{n}-s\right)}+\mathrm{a}\right. \text { finite expression, }
$$

end

$$
P(s, \lambda)=\frac{\left(-s_{n}\right)^{n}}{\lambda \Gamma(n+1)} \frac{1}{s_{n}-s}+a \text { finite expression. }
$$

Now

$$
\Gamma(k \lambda s+k)=\Gamma\left[-n+k \lambda\left(s-s_{n}\right)\right]
$$

SER. 2. VoL. 7. No. 1019. 
has a simple pole at the point $s=s_{n}$, with the residue

$$
\frac{1}{k \lambda} \frac{(-1)^{n}}{\Gamma(n+1)} \text {. }
$$

Hence the general branch of the function $k(\Gamma k \lambda s+k) s^{-k \lambda s-k}$, in the vicinity of $s=s_{n}$, is equal to

$$
\begin{aligned}
\frac{(-1)^{n}}{\lambda \Gamma(n+1)} \frac{1}{s-s_{n}} & \exp \left[-\left(k \lambda s_{n}+k\right)\left(\log s_{n}+2 p \pi \iota\right)\right]+\text { a finite expression } \\
= & \frac{(-)^{\prime \prime}}{\lambda \Gamma} \frac{\exp \left[n\left(\log s_{n}+2 p \pi \imath\right)\right] \frac{1}{s-s_{n}}+\text { a finite expression }}{(n+1)} \operatorname{l2} \\
= & \frac{\left(-s_{n}\right)^{n}}{\lambda \Gamma(n+1)} \frac{1}{s-s_{n}}+a \text { finite expression. }
\end{aligned}
$$

Adding (1) and (2) we see that $S(s)$ is finite at $s=s_{n}$ and has no singularity there.

There is therefore no finite singularity other than $s=0$.

4. When $\lambda$ is not zero the singularity at $s=0$ is of a complicated nature, being at once a branch-point and transcendental. If, however, $\lambda=0$, in which case the Dirichlet series is $\sum_{n=1}^{\infty} \exp \left(-s n^{1 / k}\right)$, we obtain a simpler expression for $S(s)$.

We have, on writing $\lambda=0$ in the formula (9) of $\$ 1$,

$$
\begin{aligned}
S(s) & =\Gamma(k+1) s^{-l}+\sum_{n=0}^{\infty} \frac{(-s)^{n}}{\Gamma(n+1)} \xi(-n / k) \\
& =\Gamma(k+1) s^{-k}-\frac{1}{2}+\sum_{n=1}^{\infty} \frac{(-s)^{n}}{\Gamma(n+1)} \xi(-n / k),
\end{aligned}
$$

since $\hat{\zeta}(0)=-\frac{1}{2}$.

The infinite series on the right-hand side of (1) is an integral function of $s$ of order $k /(k-1)$.

This result is easily established as follows.

We have $\quad \zeta(-n / k)=-2(2 \pi)^{-n k-1} \sin (n \pi / 2 k) r(1+n / k) \zeta(1+n / k)$.

Now $\zeta(1+n / k)=1+\epsilon(n)$, since $\lim _{n \rightarrow x} \zeta(1+n / k)=1$.

Therefore

$$
\begin{aligned}
& \begin{array}{l}
S(-n ! k \\
\Gamma(n+1)
\end{array}<K \frac{\Gamma(1+n / k)}{\Gamma(n+1)}, \\
& \mid \begin{array}{l}
\Gamma(-n / k) \\
\Gamma(n+1)
\end{array}>K^{-1}(2 \pi)-n k \frac{\Gamma(1+n j k)}{\Gamma(n+1)},
\end{aligned}
$$


where (2) holds for all values of $n$, and (3) for all values of $n$ for which $n \pi / 2 k$ differs from every multiple of $\pi$ by more than some assigned constant, say $\pi / 4 k$. There will clearly be an infinity of values of $n$ satisfying this condition, since $2 . \pi / 4 k+\pi / 2 k<\pi$.

Employing Stirling's theorem, we obtain from (2),

$$
\left|\begin{array}{c}
\zeta(-n / k) \\
\Gamma(n+1)
\end{array}\right|<\frac{1}{n^{[(k-1 / k)-0] n}}
$$

when $n$ is sufficiently great, and from (3),

$$
\left|\frac{\zeta(-n / k)}{\Gamma(n+1)}\right|>\frac{1}{n^{((k-1 / k)+\cdots] n}}
$$

when $n$ is sufficiently great. From these inequalities it follows, by a well-known result in the theory of integral functions, that $\Sigma(-s)^{n} \frac{\zeta(-n / k)}{\Gamma(n+1)}$ is of (apparent) order $k /(k-1)$.

If $k$ is an integer, the function $S(s)$ is clearly uniform, its only finite singularity being a pole of order $k$ at the origin. If $k$ is not an integer, the origin is a branch-point.

5. We shall now study another generalisation of the series $\Sigma e^{-s n^{1 k}}$, the Dirichlet series

$$
\sum_{n=1}^{\infty} \exp \left[-s\left\{n^{1 / k}+\sum_{\nu=1}^{\mu} \rho_{\nu} n^{1 / k_{\nu}}\right\}\right],
$$

where $1<k<k_{1}<\ldots<k_{\mu}$, and where the $\rho$ 's are any complex numbers. The series defines an analytic function in the half plane determined by $>0$.

For this case we obtain the following results.

(i.) The function $S(s)$ is

$$
\psi(s)-\frac{1}{2}+Q(s)
$$

where $Q(s)$ is an integral function of $s$ of order $k /(k-1)$, with the origin for a simpie zero, and where

$$
\psi(s)=k s^{-k} \sum_{m=0}^{\infty}\left[(-)^{m} \sum_{\Sigma a_{\nu}=m}\left\{\prod_{\nu=1}^{\mu}\left(\frac{\rho_{\nu}^{a_{\nu}}}{\Gamma\left(a_{\nu}+1\right)}\right) \Gamma\left(\sum_{\nu=1}^{\mu} k a_{\nu} / k_{\nu}+k\right) s^{\hat{\Sigma}\left(1-k ; k_{\nu}\right) a_{\nu}}\right\}\right],
$$

the $\Sigma$ insiäe the square brackets being taken over all positive or zero integral values of $\alpha_{1}, a_{2}, \ldots, a_{\mu}$, such that $a_{1}+a_{2}+\ldots+a_{\mu}=m_{0}$

(ii.) $S(s)$ has $s=0$ for its sole finite singularity. 
(iii.) If a cut be made along the negative real axis, and if $S_{0}(s)$ be the function which is the continuation of the Dirichlet series in the cut plane, we have

$$
S_{0}(s)=\psi_{0}(s)-\frac{1}{2}+Q(s),
$$

where $\psi_{0}(s)=\psi(s)$, with the restriction that every term $s^{\lambda}$ appearing in $\psi(s)$ is to be interpreted as $\exp (\lambda \log s)$, log s having its principal value.

(iv.)* If the above expression for $\psi(s)$ be arranged in order of nondecreasing indices of $s$, each term of the old expression being retained as a separate term of the new one, we have

$$
\psi(s)=s^{-k}\left[\Gamma(k+1)+\sum_{n=1}^{\infty} c_{n} s^{\theta_{n}}\right],
$$

where $0<\theta_{1} \leqslant \theta_{2} \leqslant \ldots$,

and

$$
\theta_{n}>K^{-1} n^{1 /(k+1)},
$$

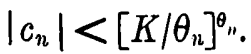

We suppose $s$ real and positive, and proceed almost exactly as in $\S 1$. We obtain a result analogous to (5) of that article, but instead of (6) we have

$$
I_{2}^{\prime \prime}+I_{3}^{\prime \prime}=-\frac{1}{2}+\epsilon(\alpha) \text {. }
$$

Thus, for $s$ real and positive,

$$
S_{0}(s)=\int_{0}^{\infty} \exp \left[-s\left\{x^{1 k}+\sum_{1}^{\mu} \rho_{\nu} x^{1, k_{\nu}}\right\}\right] d x-\frac{1}{2}+Q(s)
$$

$$
\left.\begin{array}{l}
\text { where } \\
Q(s)=\mathfrak{R} 2 \sum_{n=1}^{\infty} \frac{(-s)^{n}}{\Gamma(n+1)} \int_{0}^{\infty}\left[t^{1 / k} e^{\frac{3 \pi \tau / k}{1 k}}+\sum_{1}^{\mu} \rho_{\nu} t^{1, k_{\nu}} e^{\frac{3 \pi r / k}{\nu}}\right]^{n}\left(e^{2 \pi t}-1\right)^{-1} d t
\end{array}\right\},
$$

and where $S_{0}(s)$ stands for the Dirichlet series.

That $Q(s)$ is an integral function of order $k /(k-1)$ is easily seen as follows.

The modulus of the coefficient of $s^{n}$ is less than

$$
\begin{aligned}
& \frac{2}{\Gamma(n+1)} \int_{0}^{\infty} \frac{\left|\left[t^{1 / k} e^{\pi * 2 k}+\sum \rho_{\nu} t^{1 / k_{\nu}} e^{\pi+/ 2 k_{\nu}}\right]^{n}\right|}{e^{2 \pi t}-1} d t<\frac{2}{\Gamma(n+1)} \int_{0}^{\infty} \frac{\left[K t^{1 / k}\right]^{n}}{e^{2 \pi t}-1} d t \\
& <\frac{2 K^{n}}{\Gamma(n+1)} \zeta(-n / k)<n-i(k-1) / k+\cdot d n,
\end{aligned}
$$

when $n$ is sufficiently great, by the proof given in $\S 4$.

Hence the apparent order of $Q(s)$ is not greater than $k /(k-1)$.

- This result is required in Part II., where we determine an asymptotio expansion for the integral function corresponding to the Dirichlet series. 
The further result, less important than the above for our later requirements, that $Q(s)$ is actually of apparent order $k /(k-1)$ may be established in the following way.

After some finite value of $t$, depending on $\epsilon$, we have

$$
\left|\sum_{1}^{\mu} \rho_{\nu} t^{l, k}\right|<\epsilon \cdot t^{1 k}
$$

It follows without difficulty that

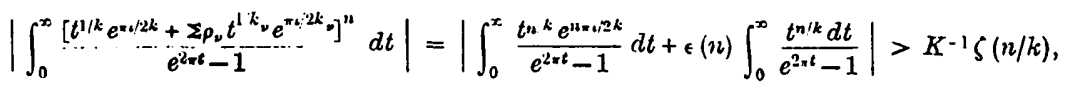

for an intinite number of values of $n$.

We have, then, for an infinite number of values of $n$,

$$
\text { | the coefficient of } s^{n} \text { in } Q(s) \mid>K^{-1} \zeta(-n / k) / \Gamma(n+1)>n-1 k-1 k+\therefore n \text {, }
$$

whence it follows that the apparent order of $Q(s)$ is not less than $k /(k-1)$, and therefore that it must actually bo $k /(k-1)$.

Returning to (1), consider the integral

$$
\int_{0}^{\infty} \exp \left[-s\left\{x^{1, k}+\Sigma_{\rho_{\nu}} x^{1, k}\right\}\right] d x .
$$

If we make the transformation $x=(y / s)^{k}$, it becomes

$$
\psi(s)=k s^{-k} \int_{0}^{\infty} \exp \left[-\sum_{\nu=1}^{\mu} \rho_{\nu} s^{1-k / k_{\nu}} y^{k k_{\nu}}\right] y^{k-1} e^{-y} d y .
$$

Now, since $k_{v}>k$, this expression defines an analytic function of $s$ whose sole finite singularity is at $s=0$. Therefore, since the Dirichlet series is equal to $\psi(s)-\frac{1}{2}+Q(s)$ when $s$ is real and positive, $\psi(s)-\frac{1}{2}+Q(s)$ must be the function obtained by continuation from the Dirichlet series.

If a cut be made along the negative real axis, since a power $s^{\wedge}$ of $s$ occurring in (2) means $\exp (\lambda \log s)$, the logarithm being real, the function $\psi_{0}(s)-\frac{1}{2}+Q(s)$ obtained by continuation in the cut plane from the Dirichlet series is $\psi(s)-\frac{1}{2}+Q(s)$, with the restriction that any power $s^{\lambda}$ occurring in the expression for $\psi(s)$ given by (2) is to mexn $\exp (\lambda \log s)$, where $\log s$ has its principal value.

We must now prove that $\psi(s)$ is given by the form in our result (i.). We have

$$
\begin{aligned}
& \exp \left[-\sum_{1}^{\mu} \rho_{\nu} s^{1-1 / k_{\nu}} y^{k / k_{\nu}}\right] \\
& =\sum_{m=0}^{\infty} \frac{1}{\Gamma(m+1)}\left[-\sum \rho_{\nu} s^{1-1 / k_{v}} y^{k_{i} ; k_{v}}\right]^{m}
\end{aligned}
$$

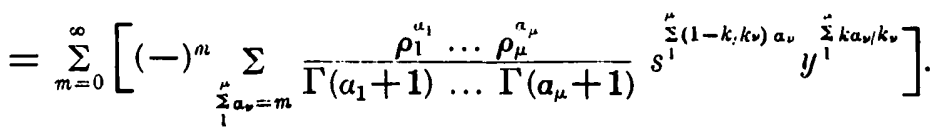


Multiplying this expression by $k s^{-k} y^{k-1} e^{-y}$, and integrating term by term from $y=0$ to $\infty$, a process justifiable without any special difficulty, we obtain

$\psi(s)=k s^{-k} \sum_{m=0}^{\infty}\left[(-)^{m} \sum_{\Sigma \alpha_{\nu}=m} \frac{\rho_{1}^{a_{1}} \ldots \rho_{\mu}^{a_{\mu}}}{\Gamma(a+1) \ldots \Gamma\left(a_{\mu}+1\right)} \Gamma\left(\Sigma k \alpha_{\nu} / k_{\nu}+k\right) s^{\Sigma\left(1-k / k_{\nu}\right) \alpha_{\nu}}\right]$,

which is the desired form.

The term corresponding to $m=0$ is $k s^{-k} \Gamma(k)$ or $\Gamma(k+1) s^{-k}$.

We have now established the results (i.), (ii.), (iii.); it remains to consider (iv.).

Let the general term in (3) be the term $s^{-k}\left(c_{n} s^{\left.\theta_{1}\right)}\right.$ in the expansion

$$
\psi(s)=s^{-k}\left[\Gamma(k+1)+\sum_{n=1}^{\infty} c_{n} s^{\theta_{n}}\right]
$$

arranged in order of increasing indices. Then

$$
\theta_{n}=\Sigma\left(1-k / k_{\nu}\right) a_{\nu}, \quad \Sigma k a_{\nu} / k_{\nu}=m-\theta_{n}
$$

and

$$
\left|c_{n}\right|<K \frac{K^{a_{1}} \ldots K^{a_{\mu}}}{\Gamma\left(a_{1}+1\right) \ldots \Gamma\left(\alpha_{\mu}+1\right)} \Gamma\left(\Sigma k \alpha_{\nu} / k_{\nu}+k\right) .
$$

Now it is easily shown that $\Gamma\left(\alpha_{1}+1\right) \ldots \Gamma\left(\alpha_{\mu}+1\right)$, when $\alpha_{1}+\alpha_{2}+\ldots+\alpha_{\mu}$ is equal to a given $m$, is a minimum when

Then

$$
\alpha_{1}=\alpha_{2}=\ldots=\frac{\Sigma \alpha_{v}}{\mu}=\frac{m}{\mu} .
$$

$$
\begin{aligned}
&\left|c_{n}\right|<K^{m+1}\{\Gamma(m / \mu+1)\}^{-\mu} \Gamma\left(\sum k a_{v} \mid k_{\nu}+k\right) \\
&<K^{m+1} \exp \left[\left\{\Sigma k a_{\nu} \mid k_{\nu}+k\right\} \log \left\{\Sigma k a_{\nu} \mid k_{\nu}+k\right\}-\left\{\Sigma k a_{\nu} / k_{\nu}+k\right\}+K\right. \\
&\left.-\mu\left(\frac{m}{\mu} \log \frac{m}{\mu}-\frac{m}{\mu}+\frac{1}{2} \log \frac{m}{\mu}-K\right)\right]
\end{aligned}
$$

(on using Stirling's theorem)

$$
<K^{m+1} \exp \left[\left(m-\theta_{n}+k\right) \log \left(m-\theta_{n}+k\right)-m \log m+K m\right] .
$$

Now

$$
m-\theta_{n}=\Sigma \frac{k \alpha_{v}}{k_{v}}<\frac{k}{k_{1}} \Sigma \alpha_{v}<\frac{k}{k_{1}} m
$$

and therefore

$$
\theta_{n}>K^{-1} m \text {. }
$$


Then, from (4), we have

$$
\left|c_{n}\right|<K^{\theta_{n}} \exp \left[\left(m-\theta_{n}\right) \log m-m \log m\right]^{*}<K^{\theta_{n}} \cdot \theta_{n}^{-m}<\left(K / \theta_{n}\right)^{\theta_{n}} .
$$

We have, finally, to prove that

$$
\theta_{n}>K^{-1} n^{1 /(\mu+1)}
$$

Now the number of terms in the expansion of

$$
\left[\sum_{1}^{\mu} \rho_{\nu} s^{1-k / k_{\nu}} y^{k / k_{\nu}}\right]^{m}
$$

is less than $K m^{\mu}$, and all the indices of $s$ which occur are greater than $m / K_{1}$. Hence the number $n$ of terms of the type $s^{\frac{5}{\Sigma}\left(1-k / k_{i}\right) a_{\nu}}$ which have an index less than $\theta_{n}$ is less than

and therefore

$$
\sum_{n=1}^{K_{1} \theta_{n}} K m^{\mu}<K \theta_{n}^{\mu+1}
$$

$$
\theta_{n}>K^{-1} n^{1 / \mu+1)} \text {. }
$$

6. In the case when $\mu=1$, the result (i.) for $\psi(s)$ becomes simpler, and we obtain for the function generated by the Dirichlet series

$$
\begin{gathered}
\sum_{n=1}^{\infty} \exp \left[-s\left(n^{1 / k}+\rho_{1} n^{1 / k_{1}}\right)\right], \\
S(s)=s^{-k}\left[\Gamma(k+1)+k \sum_{n=1}^{\infty} \frac{\left(-\rho_{1}\right)^{n} s^{n\left(1-k / k_{1}\right)}}{\Gamma(n+1)} \Gamma\left(n k / k_{1}+k\right)\right] \\
-\frac{1}{2}-\left\{\zeta(-1 / k)+\rho_{1} \xi\left(-1 / k_{1}\right)^{\prime} s+s^{2} Q_{1}(s),\right.
\end{gathered}
$$

where $Q_{1}(s)$ is an integral function of order $k /(k-1)$ and where the series in square brackets is an integral function of the argument $s^{1-k / k_{1}}$ of order $k /\left(k_{1}-k\right)$.

7. We have so far cunsidered Dirichlet series for which $\left|a_{n}\right|$ is of order $\exp \left(n^{\rho}\right)$ for some value of $\rho$ less than unity. The simplest type of function which is of less order than $\exp \left(n^{\rho}\right)$ for all values of $\rho$, however small, but of greater order than $n^{\rho}$ for all values of $\rho$, however large, is

where $k$ is positive. 1

$$
\exp \left[(\log n)^{1+k}\right] \text {, }
$$

* We have

$K^{m+1} \exp \left[k \log \left(m-\theta_{n}+k\right)+K m\right]<K^{m+1} \exp (K m)<K^{(m+1)(1+\log K)}<K^{m}<K^{K \theta_{n}}<K_{\theta_{n}}$, according to our use of the symbol $K$.

$\dagger$ There exists, of course, an infinity of types, intcrmediate in order between exp $\left(n^{\circ}\right)$ and $\exp \left[(\log n)^{1+k}\right] . \quad \exp \left[\exp (\log n)^{1, i k+1}\right]$ is in eximple. 
Let us consider, then, the Dirichlet series

$$
\sum_{n=2}^{\infty} \exp \left[-s(\log n)^{1+k}\right] \text {. }
$$

I shall dwell only on points where the analysis differs in character from that already employed in other cases.

We start with the integral

$$
\int_{L_{1}+I_{2}} \exp \left[-s(\log x)^{1+k}\right] \frac{d x}{e^{2 \pi \pi x_{1}}-1},
$$

in which $s$ is real and positive, $\log x$ is supposed to have its principal value, and where $L_{1}+L_{2}$ is the contour consisting of the straight lines $+\infty+\imath \beta$ to $1+\alpha+\imath \beta$ to $1+\alpha-\imath \beta$ to $+\infty-\imath \beta, \beta$ being any positive number, and $a$ being any number between 0 and 1 .

The integral has the value $S(s)$.

Following the lines of $\$ 1$, we obtain

where

$$
S(s)=I_{1}+I_{2}+I_{8}
$$

Now

$$
\left.\begin{array}{l}
I_{1}=-\int_{L_{1}} \exp \left[-s(\log x)^{1+k}\right] d x, \\
I_{2}=\int_{L_{1}} \exp \left[-s(\log x)^{1+k}\right] \frac{d x}{1-e^{-2 \pi x_{1}}} \\
I_{3}=\int_{L_{2}} \exp \left[-s(\log x)^{1+k}\right] \frac{d x}{e^{2 \pi x_{1}}-1}
\end{array}\right\} .
$$

$$
I_{1}=\int_{1+a}^{\infty} \exp \left[-s(\log x)^{1+k}\right] d x .
$$

Putting $s(\log x)^{1+k}=y$, we have

$$
\begin{aligned}
I_{1} & =\int_{s[\log (1+a)]^{1+k}}^{\infty} e^{-y} \exp \left[(y / s)^{1 /(1+k)}\right] s^{-1(1+k)}(1+k)^{-1} y^{-k /(1+k)} d y \\
& =\frac{s^{-1 /(1+k)}}{1+k} \int_{0}^{\infty} e^{-y} \exp \left[(y / s)^{1 /(1+k)}\right] y^{-k /(1+k)} d y+\epsilon(a),
\end{aligned}
$$

since the integral is convergent at $y=0$.

The contours $L_{1}, L_{2}$ in $I_{2}$ and $I_{3}$ may be deformed into $M_{1}$ and $M_{2}$ respectively, where $M_{1}$ consists of the straight line from $1+\imath \infty$ to $1+\iota a$, together with the quadrant of the circle $|x-1|=\alpha$, from $1+\iota a$ to $1+\alpha$, and where $M_{2}$ is the image of $M_{1}$ in the real axis.

We divide $I_{2}$ and $I_{8}$ into $I_{2}^{\prime}, I_{2}^{\prime \prime} ; I_{8}^{\prime}, I_{3}^{\prime \prime}$, corresponding to the straight and curved parts of their respective contours. Then it is easily seen that

$$
I_{2}^{\prime \prime}=\int_{1+\alpha,}^{1+a} \exp \left[-s(\log x)^{1+k}\right] \frac{d x}{1-e^{-2 \pi x i}}=-\frac{1}{4}+\epsilon(\alpha),
$$


and

whence

$$
\begin{gathered}
I_{3}^{\prime \prime}=-\frac{1}{4}+\epsilon(a), \\
I_{2}^{\prime \prime}+I_{3}^{\prime \prime}=-\frac{1}{2}+\epsilon(a) .
\end{gathered}
$$

The expression $I_{2}^{\prime}+I_{3}^{\prime}$ may be transformed as in $\S 1$. Putting $x=1+t e^{ \pm \frac{1 \pi}{2}}$ on $M_{1}$ and $M_{2}$ respectively, we obtain

$$
\begin{aligned}
I_{2}^{\prime}+I_{3}^{\prime} & =\sum_{n=1}^{\infty} \frac{(-s)^{n}}{n !}\left[2 \mathfrak{A R} \int_{a}^{\infty}\left\{\log \left(1+t e^{\frac{\hbar}{3} \iota}\right)\right\}^{(1+k) n} \frac{\iota d t}{e^{2 \pi t}-1}\right] \\
& =\sum_{n=1}^{\infty} \frac{(-s)^{n}}{n !}\left[2 \mathfrak{a R} \int_{0}^{\infty}\left\{\log \left(1+t e^{\xi \pi t}\right)\right\}^{(1+k) n} \frac{\iota d t}{e^{2 \pi t}-1}\right]+\epsilon(\alpha),
\end{aligned}
$$

all the integrals being convergent at the origin."

From (1), (2), (3), and (4) we now have, when $s$ is real and positive,

and therefore

$$
S(s)=\psi(s)-\frac{1}{2}+P(s)+\epsilon(u),
$$

$$
\begin{aligned}
& S(s)=\psi(s)-\frac{1}{2}+P(s), \\
& \text { where } \psi(s)=\frac{s^{-1 /(1+k)}}{1+k} \int_{0}^{\infty} \exp \left[s^{-1 /(1+k)} y^{1 /(1+k)}\right] e^{-y} y^{-k /(1+k)} d y \\
& \left.P(s)=\sum_{n=1}^{\infty} \frac{(-s)^{n}}{n !}\left[2 \mathfrak{R} \int_{0}^{\infty}\left\{\log \left(1+t e^{3 \pi n}\right)\right\}^{(1+k) n} \begin{array}{cc} 
& \\
& e^{2 \pi t}-1
\end{array}\right]\right\} \text {, }
\end{aligned}
$$

Now, for the coefficient $c_{n}$ of $s^{n}$ in $P(s)$, we have

$$
\left|c_{n}\right|<\frac{1}{n !} \int_{0}^{\infty}\left(K t^{t}\right)^{(1+k) n} \frac{d t}{e^{2 \pi t}-1} \quad\left[\text { since }\left|\log \left(1+t e^{3 \pi}\right)\right|<K t^{t}\right],
$$

and, by a slight modification of the proof given in $\S 5$, it may be shown that $P(s)$ is an integral function of order unity.

The expression for $\psi(s)$ defines an analytic function regular at every point except $s=0$. Then $\psi(s)-\frac{1}{2}+P(s)$, since it agrees in value with the Dirichlet series when $s$ is real and positive, must be the analytic function obtained by continuation from the Dirichlet series.

If we expand the term $\exp \left[s^{-1(1+k)} y^{1 /(1+k)}\right]$ in the expression for $\psi(s)$ in (5), multiply by $(1+k)^{-1} s^{-1 /(1+k)} e^{-y} y^{-k /(1+k)}$, and integrate term by term from $y=0$ to $\infty$, we obtain

$$
\begin{aligned}
\psi(s) & =(1+k)^{-1} s^{-1 /(1+k)} \sum_{n=0}^{\infty} \frac{s^{-n /(1+k)}}{\Gamma(n+1)} \int_{0}^{\infty} e^{-y} y^{(n+1) /(1+k)-1} d y \\
& =(1+k)^{-1} \sum_{n=0}^{n} s^{-(n+1),(1+k)} \frac{\Gamma\{(n+1) /(1+k)\}}{\Gamma(n+1)} .
\end{aligned}
$$

- The legitimacy of this step may be established without difficulty. 
Thus $\psi(s)$ is an integral function of the argument $s^{-1 / 1+k)}$ of order $(k+1) / k$.

8. It is not difficult to see that the more general Dirichlet series

$$
\sum_{n=2}^{\infty} \exp \left[-s\left\{(\log n)^{1+k}+\sum_{\nu=1}^{\mu} \rho_{\nu}(\log n)^{1+k_{\nu}}\right\}\right]
$$

where $k>k_{1}>k_{2}>\ldots$, and where the $\rho$ 's are any complex numbers, gives rise to an analytic function $S(s)$ whose sole finite singularity is at $s=0$.

Proceeding as in the last article, we find that, when $s$ is real and positive,

$$
S(s)=\psi(s)+P(s),
$$

where $P(s)$ is an integral function of order unity, and

$$
\psi(s)=\int_{1}^{\infty} \exp \left[-s\left\{(\log x)^{1+k}+\sum_{1}^{\mu} \rho_{\nu}(\log x)^{1+k_{\nu}}\right\}\right] d x .
$$

On putting $x=\exp \left[(y / s)^{1 /(1+k)}\right]$, the last expression becomes $\frac{s^{-1 /(1+k)}}{1+k} \int_{0}^{\infty} \exp \left[-\sum_{1}^{\mu} \rho_{\nu} s^{\left(k-k_{\nu}\right) /\left(1+k_{\nu}\right)} y^{(1+k) /(1+k)}\right] \exp \left[s^{-1 /(1+k)} y^{1 /(1+k)}\right] e^{-y} y^{-k /(1+k)} d y$, which defines an analytic function regular at all points except $s=0$.

The function $S(s)$, then, is $\psi(s)+P(s)$, which has $s=0$ for its sole finite singularity.

9. As an example in which $a_{n}$ is near its lower limit of order in $n$, let us consider the series

$$
\sum_{n=3}^{\infty} \exp [-s \log n \log \log n] .
$$

Here $a_{n}$ is the $n$-th zero of an integral function of zero order, but $\left|a_{n}\right|$ is of less order in $n$ than $\exp \left[(\log n)^{1+k}\right]$, the case last considered, for all positive values of $k$.

We obtain, on the lines previously developed, $s$ being supposed real and positive,

$$
\text { where } \left.\quad \begin{array}{rl}
S(s) & =\int_{e+a}^{\infty} \exp [-s \log x \log \log x] d x+I^{\prime}+I^{\prime \prime}, \\
I^{\prime} & =\int_{e+a}^{e+a+\infty} \exp [-s \log x \log \log x] \frac{\iota d x}{e^{-2 \pi x}-1} \\
I^{\prime \prime} & =\int_{e+a}^{e+a-\infty \infty} \exp [-s \log x \log \log x] \frac{-\imath d x}{e^{2 \pi x t}-1}
\end{array}\right\},
$$

and where $0<a<3-e$. 
1908.] ASYMPTOTIC EXPANSIONS OF INTEGRAL FUNCTIONS OF ZERO ORDER. 235

Then

$$
\begin{aligned}
S(s)= & \int_{1}^{\infty} \exp [-s \log x \log \log x] d x \\
& -\int_{1}^{e+a} \exp [-s \log x \log \log x] d x+I^{\prime}+I^{\prime \prime} .
\end{aligned}
$$

Now each of the last three terms defines a function of $s$, regular at every finite point, and therefore the three terms together define such a function, i.e., an integral function $P(s)$ of $s$. The order of this function may be shown to be unity.

Again, if we put $\log x=y / s$,

$$
\begin{aligned}
\int_{1}^{\infty} \exp [-s \log x \log \log x] d x & =\int_{0}^{\infty} \exp [-y(\log y-\log s)] \exp (y / s) d(y / s) \\
& \left.=s^{-1} \int_{0}^{\infty} \exp \left[\log s+s^{-1}\right) y\right] e^{-y \log y} d y \\
& =s^{-1} \sum_{n=0}^{\infty} \frac{\left(\log s+s^{-1}\right)^{n}}{\Gamma(n+1)} \int_{0}^{\infty} e^{-y \log y} y^{n} d y \\
& =s^{-1} T\left(\log s+s^{-1}\right)
\end{aligned}
$$

where $T(x)$ may be shown to be an integral function of infinite order.*

The analytic function $s^{-1} T\left(\log s+s^{-1}\right)$ has $s=0$ for its sole finite singularity, and we see that the function $S(s)$ is $P(s)+s^{-1} T\left(\log s+s^{-1}\right)$ and has $s=0$ for its sole finite singularity.

[It may be shown in a somewhat similar manner that the function $S(s)$, defined by the series $\sum_{n=h}^{\infty} \exp \left[-s \log n\left(\log _{p} n\right)^{k}\right]$, has $s=0$ for its sole finite singularity, where $k>0, \log _{r} n$ stands for $\log [\{\log \ldots ; n]$ ( $p$ logarithms), and where $h$ is so large that $\log _{p} h>0$.] †

* It may be shown that $|T(x)|<\exp \exp [(1+\epsilon)|x|]$, when $|x|$ is sufficiently grcat. It follows that, for sufficiently large values of $|s|$,

and therefore

$$
\left|s^{-1} T\left(\log s+s^{-1}\right)\right|<\exp \left(|s|^{1+0}\right) \text {, }
$$

$$
|S(s)|<\exp \left(|s|^{1+c}\right) \text {. }
$$

When $|s|$ is small, however, we have the inequality,

$$
|S(s)|<\exp \exp \left[(1+\epsilon)|s|^{-1}\right],
$$

when $|s|$ is sufficiently small.

$\dagger$ [Added February 3rd.] 
PART II.

\section{Asymptotic Expansions.}

1. In the present part it will be shown how our knowledge of the function $S(s)$ enables us, in certain cases, to determine an asymptotic expansion for the corresponding integral function. We must, however, for reasons given in the Abstract, first establish the following general theorem :-

Let $F(z)$ be an analytic function, with a finite number of singularities, and possessing the following properties :-

(1) In the region $R$ consisting of the whole of the plane on one side of a straight line $L$, and including the points of $L$, a branch $F_{0}(z)$ of $F(z)$ has no singularities, and for all points of $R$,

$$
\left|F_{0}(z)\right|<K \exp \left(r^{p-1+e}\right)
$$

where $\rho$ is a positive constant greater than unity, $r=|z|$, and $\epsilon$ is any arbitrary positive number.

(2) In the whole plane, assuming the singularities to be excluded by small circles of radius $\delta,|F(z)|<K \exp \left(r^{p+\varepsilon}\right)$

for any arbitrary $\epsilon$.

Let $L^{\prime}$ be the straight line parallel to $L$ and at a distance $l$ from it on the side of $L$ remote from $R$, and let $R^{\prime}$ be the region of the plane on the same side of $L^{\prime}$ as $R$.

Let a system of cuts be made so as to prevent the point $z$, when it is restricted to the region $R^{\prime}$, from circumscribing any singularity of $F(z)$, so that $F_{0}(z)$, when continued over $R^{\prime}$, generates a uniform function.

Then, for any arbitrary $\epsilon$, we shall have for all points of $R^{\prime}$,

$$
\left|F_{0}(z)\right|<K \exp \left(p^{p-1+\bullet}\right) \text {. }
$$

Take any fixed point $P$ of $L$, and a line $\Lambda$ passing through $P$ making an angle with $L$ less than $\frac{1}{4} \pi$. Let $\Gamma$ be a circle with $P$ as centre including all the sin-

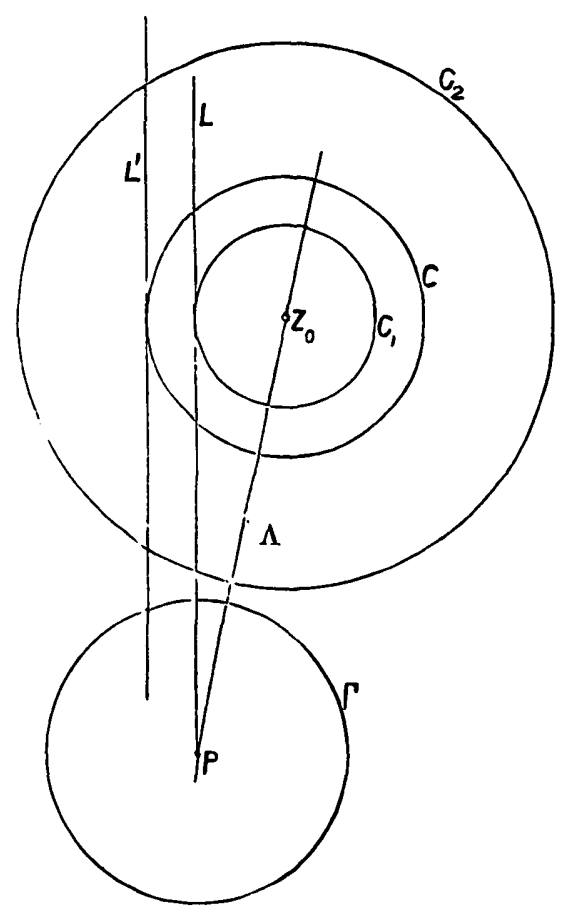
gularities of $F(z)$. 
Let $z_{0}$ be a point of $\Lambda$ lying in $R$, and such that $\left|z_{0}\right|=r_{0}$ is large. Let $C_{1}$ be the circle with $z_{0}$ as centre, touching $L$, and let $\lambda_{1} \%_{0}$ be its radius, so that $\lambda_{1}<1-K^{-1}$.* Let $C_{2}$ be the circle with $z_{0}$ as centre, and with radius $\lambda_{2} r_{0}$, where $\lambda_{1}<\lambda_{2}<1$. When $\left|z_{0}\right|$ is large, the circle $C_{2}$ will be exterior to $\Gamma$ (since $\lambda_{2}<1$ ). Let $C$ be the circle with $z_{0}$ as centre, touching $L^{\prime}$.

Now no point of $C_{1}$ is exterior to $R$. Therefore, for all points of $C_{1}$,

$$
\left|F_{0}(z)\right|<K \exp \left(r^{\rho-1+\epsilon}\right)<K \exp \left[\left(1+\lambda_{1}\right) r_{0}\right]^{p-1+\epsilon},
$$

or, since $\epsilon$ is arbitrary, $\left|F_{0}(z)\right|<K \exp \left(\gamma_{0}^{p-1+e}\right)$.

Consequently, if $\sum_{n=0}^{\infty} c_{n}\left(z-z_{0}\right)^{n}$ be the expansion of $F_{0}(z)$ about the point $z_{0}$, we have, for points $z$ of $C_{1}$,

$$
\begin{aligned}
\left|c_{n}\left(z-z_{0}\right)^{n}\right| & <\left[\text { the maximum modulus of } F_{0}(z) \text { on } C_{1}\right] \\
& <K \exp \left(r_{0}^{\rho-1+e}\right)
\end{aligned}
$$

or $\quad\left|c_{n}\right|\left(\lambda_{1} r_{0}\right)^{n}<K \exp \left(r_{0}^{\rho-1+e}\right)$

where, what is important to notice, $K$ is independent of both $n$ and $r_{0}$.

Again, $C_{2}$ contains no singularity of $F(z)$, and thus lies within the circle of convergence of $\Sigma c_{n}\left(z-z_{0}\right)^{n}$. Moreover, we have for points $z$ of $C_{2}$,

$$
\left|F_{0}(z)\right|<K \exp \left(r^{\rho+e}\right)<K \exp \left[\left\{\left(1+\lambda_{2}\right) r_{0}\right\}^{\rho+e}\right]
$$

so that

$$
\left|F_{0}(z)\right|<K \exp \left(\vartheta_{0}^{p+e}\right) \text {. }
$$

Hence we have

or

$$
\begin{gathered}
\left|c_{n}\left(z-z_{0}\right)^{n}\right|<K \exp \left(\gamma_{0}^{p+e}\right), \\
\left|c_{n}\left(\lambda_{2} r_{0}\right)^{n}\right|<K \exp \left(r_{0}^{p+e}\right)
\end{gathered}
$$

where $K$ is independent of both $n$ and $r_{0}$.

From (1) and (2) we now deduce the following result

$$
\left|c_{n}\right|<K \exp \left(r_{0}^{\rho-1+\epsilon}\right)\left(\lambda_{1} r_{0}+l+1\right)^{-n}
$$

where $K$ is independent of both $n$ and $r_{0}$.

\footnotetext{
- That is, when $r_{0}$ is large. For the origin is at a flxed distance, independent of $r_{0}$, from $P$.
} 
We have

$$
\begin{aligned}
& \exp \left(r_{0}^{\left.p-1+\frac{t^{e}}{}\right)}\right)\left(\lambda_{1} r_{0}\right)^{-n} /\left[\left(\lambda_{1} r_{0}+l+1\right)^{-n} \exp \left(r_{0}^{p-1+\varepsilon}\right)\right] \\
& =\left[\left\{1+\frac{l+1}{\lambda r_{0}}\right\}^{\lambda r_{0}}\right]^{n /\left(\lambda r_{0}\right)} \exp \left[-r_{0}^{p-1+e}+r_{0}^{p-1+l e}\right] \\
& <\left[e^{K}\right]^{n /\left(\lambda r_{0}\right)} \exp \left[-r_{0}^{\rho-1+e}+r_{0}^{\rho-1+\frac{b^{c}}{e}}\right] \text {; }
\end{aligned}
$$

and therefore, provided $n \leqslant r_{0}^{\rho+\frac{k}{2} e}$,

$$
<\exp \left[-r_{0}^{\rho-1+e}+K r_{0}^{\rho-1+\frac{t}{2} e}\right]<K .
$$

Therefore, when $n \leqslant i_{0}^{\rho+k e}$,

$$
\exp \left(r_{0}^{p-1+1}\right)\left(\lambda_{1} r_{0}\right)^{-n}<K\left(\lambda_{1} r_{0}+l+1\right)^{-n} \exp \left(r_{0}^{\rho-1+\varepsilon}\right) .
$$

Again,

$$
\begin{aligned}
\left(\lambda_{2} r_{0}\right)^{-n} \exp \left(r_{0}^{\rho+t \epsilon}\right) /\left[\left(\lambda_{1} r_{0}+l\right.\right. & \left.+1)^{-n} \exp \left(r_{0}^{\rho-1+\epsilon}\right)\right] \\
& <\left[\frac{\lambda_{1}}{\lambda_{2}}+\frac{K}{r_{0}}\right]^{n} \exp \left(r_{0}^{\rho+1 \epsilon \epsilon}\right) \\
& <\exp \left[-n \log \left(\frac{\lambda_{2}}{\lambda_{1}}\right)+\frac{K n}{r_{0}}+r_{0}^{\rho+1 e}\right] .
\end{aligned}
$$

Now, provided $n>r_{0}^{\rho+\frac{1}{2} e}$, the term $-n \log \left(\lambda_{2} / \lambda_{1}\right)$ is of higher order in $r_{0}$ than $K n / r_{0}+r_{0}^{\rho+\rfloor \epsilon}$, and therefore

$$
\exp \left[-n \log \left(\frac{\lambda_{2}}{\lambda_{1}}\right)+\frac{K n}{r_{0}}+r_{0}^{p+t e}\right]<K .
$$

We bave then, when $n>r_{0}^{p+k e}$,

$$
\left(\lambda_{2} r_{0}\right)^{-n} \exp \left(r_{0}^{\rho+\frac{1}{f}}\right)<K\left(\lambda_{1} r_{0}+l+1\right)^{-n} \exp \left(r_{0}^{\rho-1+e}\right) .
$$

If now, in (1), we replace $\epsilon$ by $\frac{1}{2} \epsilon$, and compare with (4), we see that the inequality (3) holds when $n \leqslant r_{0}^{++h e}$.

Again, replacing $\epsilon$ by $\frac{1}{4} \epsilon$ in (2), and comparing with (5), we see that (3) holds when $n>r_{0}^{\rho+h e}$.

The inequality (3) holds, then, in any case.

It is now easy to deduce our theorem.

For all points $z$ upon or within the circle $C$, or $\left|z-z_{0}\right|=\lambda_{1} r_{0}+l$, we have

$$
\begin{aligned}
\left|F_{0}(z)\right| & \leqslant \sum_{0}^{\infty}\left|c_{n}\right|\left|z-z_{0}\right|^{n} \\
& \leqslant \sum_{0}^{\infty}\left|c_{n}\right|\left(\lambda_{1} r_{0}+l\right)^{n} \\
& <K \exp \left(r_{0}^{\rho-1+e}\right) \sum_{0}^{\infty}\left(\frac{\lambda_{1} r_{0}+l}{\lambda_{1} r_{0}+l+1}\right)^{n} \\
& <K \exp \left(r_{0}^{\rho-1+e}\right)\left(\lambda_{1} r_{0}+l+1\right) .
\end{aligned}
$$


Now

$$
r>\left(1-\frac{\lambda_{1} r_{0}+l}{r_{0}}\right) r_{0}>\left(1-\lambda_{1}-\frac{l}{r_{0}}\right) r_{0} .
$$

Therefore, since $\lambda_{1}<1$,

$$
\exp \left(r_{i i}^{p-1+e}\right)\left(\lambda_{1} r_{0}+l+1\right)<K \exp \left(r^{p-1+2 e}\right),
$$

and

$$
\left|F_{0}(z)\right|<K \exp \left(p^{\infty-1+2 \epsilon}\right) .
$$

Since $\epsilon$ is arbitrary, we have, then,

$$
\left|F_{0}(z)\right|<K \exp \left(\gamma^{p-1+\epsilon}\right) .
$$

This last inequality, then, holds for all points $z$ which can be internal to a circle $C$.

Again, if we take a line $\Lambda^{\prime}$ through $P$, the image of $\Lambda$ in $L$, the inequality (6) will hold for all points which can be internal to a circle $C^{\prime}$, which has its centre at a sufficiently remote point of $\Lambda^{\prime}$ lying in $R$, and which touches $L^{\prime}$.

Now every point of the strip between $L$ and $L^{\prime}$, which is exterior to some circle $\Gamma_{1}$ with its centre at $P$, can be interior to a circle $C$ or $C^{\prime}$ : and therefore (6) holds for all points of the strip exterior to $\Gamma_{1}$.

But for all points of $\Gamma_{1}$, when the singularities of $F(z)$ are excluded by small circles, the inequality (b) obviously holds, and it holds. by hypothesis. within the region $R$. It must therefore hold within the region $R^{\prime}$.

2. When, from our knowledge of the function $S(s)$, we attempt to determine an asymptotic expansion for $\log F(z)$, the problem is greatly simplified if $S(s)$ is known to be a uniform function of $s$, for in this event we can employ the ordinary theory of residues, which is inapplicable when $S(s)$ is multiform.

Now, if we refer to the various cases of Dirichlet series, considered in Part I., it will be seen that the only case in which $S(s)$ is found to be a uniform function occurs when $a_{n}=\exp \left(n^{1 / k}\right)$, where $k$ is an integer.

We shall, then, first consider this case. The following result will be established.

Let $k$ be an integer not less than 3 , and let $S(s)$ be the function clerived from the series $\sum_{n=1}^{\infty} \exp \left(-s n^{1 i}\right)$.

Let $F(z)=\prod_{n=1}^{\infty}\left[1+z / \exp \left(\rho n^{1 /}\right)\right]$, where $\mathfrak{k} \rho>0$.

Then, if $\delta$ be a positive number as small as we please, and if : be exterior to the region between the spirals defined by

$$
z=-\exp \left(\rho x \pm \delta_{\iota}\right),
$$


where $x$ takes all real values from $-\infty$ to $\infty$, we have the asymptotic expansion,

$$
\begin{aligned}
\log F(z)=\frac{(\log z)^{k+1}}{(k+1) \rho^{k}} & +\frac{2}{(k+1) \rho^{k}} \sum_{k=1}^{\kappa}{ }_{k+1} C_{2 n}\left(2^{2 n-1}-1\right) \pi^{2 n} B_{2 n}(\log z)^{k+1-2 n} \\
& -\frac{1}{2} \log z-\rho \xi(-1 / k)+\sum_{m=1}^{N}(-)^{m-1} \frac{S(-\rho m)}{m} z^{-m}+J_{N},
\end{aligned}
$$

where $\kappa=\frac{1}{2} k$ or $\frac{1}{2}(k+1)$ according as $k$ is even or odd, the B's are Bernoulli's numbers, and

$$
\lim _{|z| \rightarrow \infty}\left|J_{N} z^{N}\right|=0 .
$$

It is clear that no asymptotic expansion can be valid over the whole extent of any region containing zeros of $F(z)$.

Now the zeros, which are of the form $-\exp \left(\rho n^{1 / k}\right)$, lie on the equiangular spiral $z=-\exp (\rho x)$, where $x$ takes all real values. Thus the two spirals $z=-\exp (\rho x \pm \delta \iota)[x=-\infty$ to $\infty]$, include between them all the zeros of $F(z)$.

In the remaining part $R$ of the plane $\log z$ is an uniform function, for $z$ cannot describe a path in $R$, starting from and returning to the same point, and circumscribing the origin. We fix the determination of $\log z$ by saying that $\log z$ is to be zero at $z=1$, and we then interpret $z^{\lambda}$ to mean $\exp (\lambda \log z)$.

Let us determine the limits of the argument of $z$ for a given modulus $r$, when $z$ is restricted to the region $R$.

Let $z=-\exp \left(\rho x^{\prime}\right)$ be the point of the circle $|z|=r$ for which $x$ is real. Then the circle meets the bounding spirals in the points $-\exp \left(\rho x^{\prime} \pm \delta_{\imath}\right)$. The determination of $\log z$ at $-\exp \left(\rho x^{\prime}+\delta_{\iota}\right)$ may be found by making $z$ describe a path in $R$ from the point $z=1$ to some point of the spiral $z=-\exp \left(\rho x+\delta_{\iota}\right)$, and then making $z$ follow this spiral up to the point in question.

We thus obtain $\quad \log z=\pi \iota+\rho x^{\prime}+\delta \iota$.

Similarly, by making $z$ describe a path in $R$ from $z=1$ to a point of the spiral $z=\exp (\rho x-\delta \iota)$, we obtain for the point $\exp \left(\rho x^{\prime}-\delta_{\iota}\right)$,

$$
\log z=\pi \iota+\rho x^{\prime}-\delta \iota .
$$

It is therefore seen that for any point $z$ of $R$ we must have

$$
\log z=\rho x+\phi \iota,
$$

where $x$ is real, and where $|\phi|<\pi-\delta$. 
We now show that, if $\left|R_{\rho} \rho\right|<K$, and if $z$ be any point of $R$, and if the points $0, \pm 1, \pm 2, \ldots$ be excluded by small circles from the range of $s$,

$$
\left|\frac{z^{s}}{\sin \pi s}\right|<K \exp [-|s| / K]
$$

where $K$ is independent of $s$, though not of $z$.

Let $\rho s=\beta+t_{\text {l, }}$ so that $|\beta|<K$. Then

$$
\begin{aligned}
\left|z^{s}\right| & =|\exp [s(\rho x+\iota \phi)]| . \\
& \leqslant \exp [\beta x+|\mathfrak{R}(s \phi \iota)|] .
\end{aligned}
$$

We have also $\quad|\sin \pi s|>K^{-1} \exp |2 R \pi s i|$.

Therefore, since $|\phi|<\pi-\delta$,

$$
\left|\frac{z^{s}}{\sin \pi s}\right|<K \exp (-|s| / K) \text {. }
$$

Now suppose that $z$ is less than unity. We then have

$$
\begin{aligned}
\log F^{\prime}(z) & =\sum_{n=1}^{\infty} \log \left[1+z / \exp \left(\rho n^{1 /}\right)\right] \\
& =\sum_{n=1}^{\infty} \sum_{t=1}^{\infty}(-)^{\ell-1} \frac{z^{\ell}}{\left.t \exp \left(t \rho n^{1}\right)^{k}\right)} ;
\end{aligned}
$$

and therefore, since this double series is a uniformly convergent series of functions, each of which is expansible in a uniformly convergent power series,

$$
\begin{aligned}
& =\sum_{t=1}^{\infty}(-)^{t-1} \frac{z^{t}}{t}\left[\sum_{n=1}^{\infty} \exp \left(-t \rho n^{1 k}\right)\right] \\
& =-\sum_{t=1}^{\infty}(-)^{t} \frac{S(\rho t)}{t} z^{t} .
\end{aligned}
$$

This series must converge when $|z|<1$.

Now consider the integral

$$
-\frac{1}{2 \imath} \int_{L_{1}+L_{8}} S(\rho s) \frac{z^{s} d s}{s \sin \pi s}
$$

where $L_{1}+L_{2}$ is the contour $+\infty+\imath \beta$ to $\alpha+\imath \beta$ to $\alpha-\imath \beta$ to $+\infty-\imath \beta$, where $\beta$ is positive, and $0<a<1$. The integrand is a uniform function of $s$ inside the contour; also the integral is convergent at infinity, since $|z|<1$, and since $S(\rho s)$ is finite on the contour. The singu-

SBR. 2. vor. 7. No. 1020. 
larities of the integrand are the points $1,2,3, \ldots$, and the sum of the residues of the integrand at these points is

$$
-\frac{1}{2 \pi \imath} \sum_{t=1}^{\infty}(-)^{t} \frac{z^{t}}{t} S(\rho t)
$$

This series is convergent and has the sum $\frac{1}{2 \pi \iota} \log F(z)$. Hence, when $z<1$,

$$
\log F(z)=-\frac{1}{2_{\iota}} \int_{L_{1}+L_{2}} S(\rho s) \frac{z^{s} d s}{s \sin \pi s} .
$$

Let $M$ be the line $\mathbb{R} \rho s=$ constant passing through the point $a$, and let $N_{1}, N_{2}$ be the parts of the circle $|s|=R$ intercepted between $M$ and $L_{1}, L_{2}$ respectively. Then on $N_{1}$ and $N_{2}$. aR $\rho s \geqslant \operatorname{li} \rho a>1 / K$, and therefore

$$
|S(\rho s)|<K \text {. }
$$

Now, if $z$ be a point within the unit circle which is also a point of the region $R$, we have, as we have seen above,

$$
\left|\frac{z^{s}}{\sin \pi s}\right|<K \exp [\beta x+|\mathfrak{R}(s \phi \imath)|] /[\exp |\pi \mathfrak{h}(s \iota)|],
$$

where

$$
\beta=\mathfrak{k} s \rho \text { and } z=\exp (\rho x+\phi \imath) .
$$

Now $\beta>0$, and since $\mathfrak{R}_{\rho}>0$ and $|z|<1$, we have $x<0$; therefore

$$
\begin{aligned}
\left|\frac{z^{s}}{\sin \pi s}\right| & <K \exp [\mid \mathfrak{k}\{s(\pi-\delta) \iota ! \mid] / \exp [|\mathfrak{R}(\pi s \iota)|] \\
& <K \exp [-\delta|\mathfrak{R}(s i)|] .
\end{aligned}
$$

On putting $s=R e^{\iota \theta}$, we therefore have

$$
\left|\int_{N_{1}} S(\rho s) \frac{z^{s} d s}{s \sin \pi s}+\right| \int_{N} S(\rho s) \frac{z^{s} d s}{s \sin \pi s} \mid<2 \int_{0}^{\pi} K \exp (-\delta R \sin \theta) d \theta
$$

which tends to zero as $R$ tends to intinity.*

It follows from this last result that we may deform the contour $L_{1}+L_{2}$ of equation (3) into $M$, since we pass over no singularities of the integrand,

- For it is less thau

$$
K \int_{-1}^{\infty} \exp (-\delta R \sin \theta) d \theta<K \int_{0}^{3 \pi} \exp \left(-\begin{array}{c}
\delta R \theta .2 \\
\pi
\end{array}\right) \cdot d \theta<K \int_{0}^{\infty} \exp (-\phi) \frac{\pi d \phi}{2 \delta R} .
$$


and we have, when $z$ is a point of $R$ within the unit circle,

$$
\log F(z)=-\frac{1}{2 \imath} \int_{M} S(\rho s) \frac{z^{s} d s}{s \sin \pi s} .
$$

We shall now show that this equation holds when $z$ is any point of the region $R$. For any such $z$, we have, for all points $s$ of the contour $M$,

$$
\left|s^{-1} S(\rho s)\right|<K,
$$

since

$$
\mathfrak{a} s=\lll \rho \alpha>0 ;
$$

and, by the result (1),

$$
\left|\frac{z^{s}}{\sin \pi s}\right|<K \exp (-|s| / K) .
$$

It follows immediately that the integral in (4) is convergent. It can also be shown without difficulty that the integral is differentiable with respect to $z$, and therefore that it defines an analytic function of $z$ in the region $R$. This function agrees in value with $\log F(z)$ in the part of $R$ within the unit circle; it must therefore represent $\log F(z)$ over the whole region $R$.

Let $M^{\prime}$ be the line $\mathbb{R} \rho s=$ constant passing through the point $-(l+1)+a$. Now, when 2 a $y>a$,

$$
|S(y)|<K<K \exp \left(|y|^{\left.1^{1(k-1)+e}\right) .}\right.
$$

Also, if $y=0$ be excluded by a small circle from the range of $y$, we have for all values of $y$,

$$
|S(y)|<K \exp \left[|y|^{k(k-1)+e}\right],
$$

since, by the results of I., $\$ 4, S(y)$ is the sum of $\Gamma(k+1) y^{-k}$, and an integral function of $y$ of order $k /(k-1)$. It therefore follows, by the theorem of II., $\$ 1$, that when

$$
\mathfrak{k} y \geqslant-(l+1)+u,
$$

we have

$$
|S(y)|<K \exp \left(|y|^{1(i-1)+e}\right) .
$$

Hence, within and upon the boundaries of the strip of the $s$-plane between $M$ and $M^{\prime}$, the origin being excluded by a small circle, we have

$$
|S(\rho s)|<K \exp \left(|\rho s|^{1 /(i-1)+\epsilon}\right) .
$$

Now, for all points of the strip, we have, by (1),

$$
\left|\frac{z^{s}}{s \sin \pi s}\right|<K \exp (-|s| / K)
$$


Since $k \geqslant 3$, we have $1 /(k-1)<1$, and we can choose $\epsilon$ so that $1 /(k-1)+\epsilon<1$. Then, within the strip,

$$
\begin{aligned}
\left|S(\rho s) \frac{z^{s}}{s \sin \pi s}\right| & <K \exp \left[-\left.|s||K+K| \rho s\right|^{1 /(k-1)+\bullet}\right] \\
& <K \exp [-|s| \mid K] .
\end{aligned}
$$

It follows immediately that $\int S(\rho s) \frac{z^{s} d s}{s \sin \pi s}$, taken along $M^{\prime}$, is convergent, and further, that the same integral taken along a straight line connecting $M$ and $M^{\prime}$ tends to zero as the position of line tends to infinity. TVe have then

$$
-\frac{1}{2} \int_{M} S(\rho s) \frac{z^{s} d s}{s \sin \pi s}+\frac{1}{2 \iota} \int_{M^{\prime}} S(\rho s) \frac{z^{s} d s}{s \sin \pi s}
$$$$
=-2 \pi \iota\left[\text { the sum of the residues of }-\frac{1}{2 \iota} S(\rho s) \frac{z^{s}}{s \sin \pi s}\right. \text { at the }
$$

$$
\text { singularities between } M \text { and } M^{\prime} \text { ]. }
$$

The singularities are at the points $0,-1,-2, \ldots,-l$. The residue of $-\frac{1}{2} \frac{z^{s} S(\rho s)}{s \sin \pi s}$ at $s=-m(m \neq 0)$, is

$$
-\frac{1}{2 \pi \iota}(-)^{m-1} \frac{S(-\rho m)}{m} z^{-m}
$$

Now consider the residue at $s=0$. Near this point we have, using the form of $S(\rho s)$ given in $\S 4$, Part I.,

$$
\begin{aligned}
-\frac{1}{2 \iota} \frac{z^{s} S(\rho s)}{s \sin \pi s}= & -\frac{1}{2 \iota \pi} \Gamma(k+1) \rho^{-k} s^{-k-2} \frac{z^{s} \pi s}{\sin \pi s}-\frac{1}{2 \pi \iota} \frac{\pi z^{s}}{s \sin \pi s}\left(-\frac{1}{2}\right) \\
& -\frac{1}{2 \iota} \frac{z^{s}}{s \sin \pi s}\left[-\rho s \xi(-1 / k)+A s^{2}+\ldots\right]
\end{aligned}
$$

The residues of the second and third terms give

$$
-\frac{1}{2 \pi \iota}\left(-\frac{1}{2} \log z\right)+\frac{\rho}{2 \pi \iota} \xi(-1 / k) .
$$


As regerds the first term, we have, when $|s|$ is small,

where

$$
\frac{\pi s}{\sin \pi s}=1+\sum_{n=1}^{\infty} A_{2 n} s^{n}
$$

$$
A_{2 n}=\frac{2\left(2^{2 n-1}-1\right) \pi^{2 n}}{2 n !} B_{2 n}
$$

Hence the residue of the first term

$$
=-\frac{1}{2 \pi \iota} \rho^{-k} \Gamma(k+1)\left\{\frac{(\log z)^{k+1}}{(k+1) !}+\sum_{n=1}^{k} A_{2 n} \frac{(\log z)^{k+1-2 n}}{(k+1-2 n) !}\right\},
$$

where $\kappa$ is $\frac{1}{2} k$ or $\frac{1}{2}(k+1)$, according as $k$ is even or odd.

We now have from (4), (5), (6), (7), and (8),

$$
\begin{aligned}
\log F^{\prime}(z) & =-\frac{1}{2 \imath} \int_{M} S(\rho s) \frac{z^{s} d s}{s \sin \pi s} \\
& =T-\frac{1}{2 \iota} \int_{M^{\prime}} S(\rho s) \frac{z^{s} d s}{s \sin \pi s},
\end{aligned}
$$

where $T$ is the expansion given at the beginning of the article, with $N$ replaced by $l$, and with the term $J_{N}$ omitted.

In order to establish our result, we have now only to show that

$$
\lim _{|z| \rightarrow \infty}\left|z^{l} \int_{M^{\prime}} S(\rho s) \frac{z^{s} d s}{s \sin \pi s}\right|=0 .
$$

Let

$$
z=\exp (\rho x+\iota \phi), \quad \rho s=\rho(-l-1+\alpha)+\iota t,
$$

where $x$ is real, and where $t$ is real when $s$ is a point of $M^{\prime}$.

Then

$$
\lim _{|z| \rightarrow \infty}\left|\int_{M^{\prime}} z^{h+s} S(\rho s) \frac{d s}{s \sin \pi s}\right|
$$

$\leqslant \lim _{|z| \rightarrow \infty} \int_{-\infty}^{\infty}|\exp [(\rho x+\iota \dot{\phi})(-1+\alpha+\iota t / \rho)]| \exp \left[K|t|^{1(k-1)+\iota}\right] \frac{K d t}{\exp |\mathbf{R}(\pi \iota s)|}$

$\leqslant \lim _{|z| \rightarrow \infty} K \int_{-\infty}^{\infty}|z|^{-1+\alpha} \exp [|2 R(\phi \iota s)|] \exp \left[K|t|^{1 /(k-1)+e}\right] \exp \left[-\pi\left|a R^{\prime}(\iota s)\right|\right] d \varepsilon(9)$

$\leqslant \lim _{|z| \rightarrow \infty} K|z|^{-1+a} \int_{-\infty}^{\infty} \exp \left[K|t|^{1 /(k-1)+e}\right] \exp [-\delta|\operatorname{Re}(s i)|] d t$,

since $|\phi|<\pi-\delta$. 
Now the integral last written is convergent, and it is independent of $z$. Also, since $\alpha<1$,

$$
\lim _{|z| \rightarrow \infty}|z|^{-1+a}=0 .
$$

We therefore have

$$
\lim _{|z| \rightarrow \infty}\left|\int_{M^{\prime}} z^{l+s} S(\rho s) \frac{d s}{s \sin \pi s}\right|=0 .
$$

3. In the last article we excluded a spiral strip of the $z$-plane, containing the zeros of $F(z)$, from the range of the variable $z$. (In the particular case when $\rho$ is real, the strip becomes the angle of the plane between two lines making an arbitrarily small angle $\delta$ with the negative real axis.) The spiral region is, as a matter of fact, unnecessarily extensive, and we shall show that the expansion of the last article is valid when the excluded region is contracted upon the curve of zeros. The expansion, in fact, is valid when the excluded region has for its boundaries (outside the unit circle) the curves defined by

$$
z=\exp \left[\rho x \pm \delta \delta_{1} \cdot x^{-(i-2)+\delta}\right],
$$

where $x$ takes all values from 1 to $+\infty$ and where $\delta$ is an arbitrarily small positive number. These curves start at the points $z=\exp (\rho \pm \delta l)$; the boundaries of the region from 0 to these points may be taken to be the spirals $z=\exp \left(\rho x \pm \delta_{\iota}\right)$.*

If $z$ be any point exterior to this region, $|z|$ being supposed greater than unity, we have

$$
z=\exp (\rho x+\iota \phi),
$$

where

$$
\pi-|\phi|>\delta x^{-(i-2)+\delta}>K^{-1}(\log r)^{-(k-2)+\delta} .
$$

Since $\pi-|\phi|$ is greater than a positive number independent of $s$, we have, as in the last article,

$$
\log F(z)=T-\frac{1}{2 \iota} \int_{M^{\prime}} S(\rho s) \frac{z^{s} d s}{s \sin \pi s},
$$

and we have only to prove that

$$
\lim _{r \rightarrow \infty}\left|z^{2} \int_{M^{\prime}} S(\rho s) \frac{z s d s}{s \sin \pi s}\right|=0 .
$$

* The strip between these spirals contains no zeros of $F(z)$, but it must be excluded from the range of $z$ in order to secure the proper determination of $\log z$, which is supposed zero at $z=1$. 
Now we still have the result (9) of the last article, viz.,

$$
\begin{aligned}
& \lim _{r \rightarrow \infty}\left|\int_{M^{\prime}} z^{l+s} S(\rho s) \frac{d s}{s \sin \pi s}\right| \\
& \leqslant \lim _{r \rightarrow \infty} K r^{-1+a} \int_{-\infty}^{\infty} \exp [|\operatorname{ar} \phi \iota s|] \exp \left[K|t|^{1,(k-1)+e}\right] \exp [-|\mathfrak{2} \pi \iota s|] d t \\
& \leqslant \lim _{r \rightarrow \infty} K r^{-1+a} \int_{-\infty}^{\infty} \exp \left[K|t|^{1 /(k-1)+\varepsilon}\right] \exp \left[-K^{-1}(\log r)^{-(k+2)+\delta}|\mathfrak{2} \iota s|\right] d t, \\
& \text { since } \quad \pi-|\phi|>K^{-1}(\log r)^{-(i-2)+\delta} .
\end{aligned}
$$

Now, when $|t|>K_{1}, \mid 2\left(s\left|>K^{-1}\right| t \mid\right.$. Also the integral last written, when taken between the limits $t= \pm K_{1}$, is less than a $K$ independent of $z$, and this $K$ when multiplied by $r^{-1+a}$ has zero as a limit when $r$ is made infinite. We therefore have

$$
\begin{aligned}
& \lim _{\rightarrow \rightarrow \infty}\left|\int_{M^{\prime}} z^{l+s} S(\rho s) \frac{d s}{s \sin \pi s}\right| \\
& \leqslant \lim _{r \rightarrow \infty} K r^{-1+a} \int_{-\infty}^{\infty} \exp \left[K_{2}|t|^{\mid /(k-1)+\varepsilon}\right] \exp \left[-K_{3}^{-1}(\log r)^{-(k-2)+\delta}|t|\right] d t \\
& \leqslant \lim _{r \rightarrow \infty}\left[K r^{-1+\alpha} \sum_{n=0}^{\infty} \frac{K_{2}^{n}}{n !} \int_{-\infty}^{\infty}|t|^{n[1 /(k-1)+\varepsilon]} \exp \left[-K_{3}^{-1}(\log r)^{-(k-2)+\delta}|t|\right] d t\right] \\
& \left.\leqslant \lim _{r \rightarrow \infty}\left[K r^{-1+a} \sum_{n=0}^{\infty} \frac{2\left[K_{2} K_{3}(\log r)^{k-2-\delta}\right]^{n}}{\Gamma(n+1)} \Gamma: n(1 / \overline{k-1}+\epsilon)\right\}\right] .
\end{aligned}
$$

The infinite series is an integral function of the argument

$$
K_{2} K_{9}(\log r)^{k-2-\delta}
$$

of order

$$
1 /[1-(1 / \overline{k-1}+\epsilon)] \text {. }
$$

Therefore

$$
\begin{aligned}
\lim _{r \rightarrow \infty} \mid \int_{M^{\prime}} z^{l+\imath} & S(\rho s) \frac{d s}{s \sin \pi s} \mid \\
& \leqslant \lim _{r \rightarrow \infty} K r^{-1+a} \exp \left[K\left\{K_{2} K_{3}(\log r)^{k-2-\delta}\right\} 1 /[1-(1 / k-1+\epsilon)]+e^{\prime}\right] .
\end{aligned}
$$

Now $\epsilon$ and $\epsilon^{\prime}$ may be so chosen that

$$
(k-2-\delta)\left[1 /\{1-(1 / \overline{k-1}+\epsilon)\}+\epsilon^{\prime}\right]<1-1 / K \quad(\text { since } k>2) .
$$


Then the right-hand side of (2) is less than or equal to

$$
\lim _{r \rightarrow \infty} K r^{-1+a} \exp \left[K(\log r)^{1-1 / K}\right]=0,
$$

and the desired result is established.*

4. If $k$ is not an integer $S(\rho s)$ is no longer a uniform function, and the ordinary theory of residues cannot be applied. We shall now consider this case, and shall investigate the more general form

$$
F(z)=\prod_{n=1}^{\infty}\left[1+z / \exp \left\{\rho\left(n^{1 / k}+\sum_{\nu=1}^{\mu} \rho_{\nu} n^{1 / k_{\nu}}\right)\right\}\right],
$$

where $k_{\mu}>k_{\mu-1}>\ldots>k_{1}>k>2, \rho_{1}, \rho_{2}, \ldots$ are real, and where we suppose, for simplicity, that $\rho$ is real and, of course, positive.

We obtain the following result :-

If the angle of the z-plane contained between the two straight lines which make an arbitrarily small angle $\delta$ with the negative real axis be excluded from the range of the variable $z$, we have the asymptotic expansion

$$
\begin{aligned}
\log F(z)= & -\rho\left[\xi(-1 / k)+\sum_{\nu=1}^{\mu} \rho_{\nu} \xi\left(-1 / k_{\nu}\right)\right] \\
& \quad-\frac{1}{2} \log z+\sum_{n=0} \frac{\rho c_{n} \sin \left(k-\theta_{n}\right) \pi}{\pi}\left(\frac{\log z}{\rho}\right)^{k-\theta_{n}+1} \\
\times & {\left[\Gamma\left(\theta_{n}-k-1\right)+2 \sum_{m=1} \Gamma\left(2 m+\theta_{n}-k-1\right) \frac{\left(2^{2 m-1}-1\right) \pi^{2 m}}{2 m !} B_{2 m}(\log z)^{-2 m}\right], }
\end{aligned}
$$

where the B's are Bernoulli's numbers and $s^{-k} \sum_{n=0}^{\infty} c_{n} s^{\theta_{n}}$ is the expansion for $\psi(s)$ given in I., $\S 5$. The infinite series is to be arranged in order of increasing indices of $1 / \log z$, and when we stop at any term, of order $(\log z)^{-p}$ say, we have $\lim _{\mid \boldsymbol{x} \rightarrow \infty}\left|R(\log z)^{p}\right|=0$, where $R$ is the remainder.

Let $R$ denote the region of the $s$-plane defined by $2 a s \geqslant-l$ (where $l$ is any positive number), with the origin excluded by a small circle, and with a cut made along the negative real axis from this circle to the boundary lik $=-l$.

* The regions over which most other known asymptotic expansions, e.g., that of Barnes's function $P_{\nu}(z)$, are valid, may be extended in the same way. 
As in I., $\S 5$, let $S_{0}(\rho s)$ denote the function, uniform in $R$, which is derived by continuation over $R$ from the Dirichlet series

$$
\sum_{n=1}^{\infty} \exp \left[-\rho s\left\{n^{1 / k}+\sum_{1}^{\mu} \rho_{\nu} n^{1 / k_{\nu}}\right\}\right]
$$

defined for $₫ s>0$. We have, by I., $\S 5$, for all points $s$, and for all branches of $S(\rho s)$,

$$
S(\rho s)=\psi(\rho s)+Q(\rho s),
$$

where $Q(x)$ is an integral function of $x$ of order $k /(k-1)$,

and

where

$$
\left.\begin{array}{rl}
\psi(\rho s) & =(\rho s)^{-k}\left[\Gamma(k+1)+\sum_{n=1}^{\infty} c_{n}(\rho s)^{\theta_{n}}\right] \\
\theta_{n} & >K^{-1} n^{1 /(\mu+1)} \\
\left|c_{n}\right| & <\left[K_{1} / \theta_{n}\right]^{\theta_{n}}
\end{array}\right\} .
$$

We shall show that for all points of $R$ we have

$$
\left.\begin{array}{rl}
\left|\psi_{0}(\rho s)\right| & <K \exp \left[|s|^{\epsilon}\right] \\
|Q(\rho s)| & <K \exp \left[|s|^{1 /(k-1)+e}\right]
\end{array}\right\} .
$$

From (2) we have, for all values of $s$ exterior to the small circle excluding the origin,

$$
|\psi(\rho s)|<K+K \sum_{n=1}^{\infty}\left[K_{1} \rho|s| / \theta_{n}\right]^{\theta_{n}} .
$$

Let $m$ be the greatest value of $n$ such that

$$
K_{1} \rho|s| / \theta_{n}>e^{-1} \text {. }
$$

Now $\left[K_{1} \rho|s| \mid \theta\right]^{\theta}$ has its maximum value for $\theta>0$ when $\theta=K_{1} \rho|s| \mid e$, and the value then is $\exp \left[K_{1} \rho|s| \mid e\right]$. Hence, from (4),

$$
\begin{aligned}
|\psi(\rho s)| & <K(m+1) \exp \left[K_{1} \rho|s| / e\right]+\sum_{n=m+1}^{\infty}\left[K_{1} \rho|s| / \theta_{n}\right]^{\theta_{n}} \\
& <\left[K \theta_{m}\right]^{\mu+1} \exp [K|s|]+\sum_{n=1}^{\infty}\left[e^{-1}\right]^{\theta_{n}} \\
& <K|s|^{\mu+1} \exp [K|s|]+\sum_{n=1}^{\infty} \exp \left[-K^{-1} n^{1 /(\mu+1)}\right] \\
& <K \exp \left[|s|^{1+e}\right],{ }^{*}
\end{aligned}
$$

since the infinite series is convergent and independent of $|s|$.

* For the method of determining inequalities such as the above by finding the maximum term of the series, see Borel, Lerons sur les series $\dot{a}$ termes positifs, p. 69. 
Now let $R^{\prime}$ be the region defined by ahs $>1$. When $s$ is real and $s>1$, we have by $\$ 5$, Part I.,

$$
\psi_{0}(\rho s)=\int_{0}^{\infty} \exp \left[-\rho s\left\{x^{1 / k}+\sum_{\nu=1}^{\mu} \rho_{\nu} x^{1 / k_{\nu}}\right\}\right] d x .
$$

Now the right-hand side defines an analytic function of $s$, uniform in the region $R^{\prime}$. This function, then, must be $\psi_{0}(\rho s)$. Hence, within $R^{\prime}$,

$$
\begin{aligned}
\left|\psi_{0}(\rho s)\right| & \leqslant \int_{0}^{\infty} \exp \left[-\mathfrak{R}(\rho s)\left|\left\{x^{1 / k}+\Sigma \rho_{\nu} x^{1 / k_{\nu}}\right\}\right|\right] d x \\
& <K \quad \text { (since } \mathbb{R} \rho s \geqslant \rho) .
\end{aligned}
$$

Again, since $Q(s)$ is an integral function of order $k /(k-1)$, we have, for all values of $s$,

$$
|Q(\rho s)|<K \exp \left[|s|^{k /(k-1)+e}\right] \text {. }
$$

Also, within $R^{\prime}$, we have

$$
\begin{aligned}
\left|S_{0}(\rho s)\right| & \leqslant \sum_{n=1}^{\infty} \exp \left[-\mathfrak{2}(\rho s)\left|\left\{n^{1 / k}+\Sigma \rho_{\nu} n^{1 / k_{\nu}}\right\}\right|\right] \\
& <K \quad \text { (since } \mathfrak{a} \rho s \geqslant \rho),
\end{aligned}
$$

and therefore

$$
\begin{aligned}
|Q(\rho s)| & \leqslant\left|S_{0}(\rho s)\right|+\left|\psi_{0}(\rho s)\right| \\
& <K .
\end{aligned}
$$

Now we see, from (5) and (5)', that we may apply the theorem of II., $\S 1$, in the case of the function $\psi_{0}(\rho s)$, and we therefore have, for all points of $R$,

$$
\left|\psi_{0}(\rho s)\right|<K \exp \left[|s|^{e}\right] .
$$

Similarly, by (6) and (6)', we may apply the same theorem in the case of $Q(\rho s)$, and we have, within $R$,

$$
|Q(\rho s)|<K \exp \left[|s|^{k(k-1)-1+e}\right] .
$$

These are the desired results (3).

Now suppose that $|\arg z|<\pi-\delta$, so that $z$ is exterior to the angle of our theorem. Then proceeding exactly as in $\S 2$, we obtain

$$
\log F(z)=-\frac{1}{2 \iota} \int_{M, \frac{2}{2 \pi}} S_{0}(\rho s) \frac{z^{s} d s}{s \sin \pi s},
$$


1908.] AsYMPtotic EXPANSIONS OF INTEGRIL FUNCTIONS OF ZERO ORDER. 251

where $M$ is the line $\mathfrak{2 R} s=1-\alpha$ and $0<\alpha<1$,

where

$$
\left.\begin{array}{rl} 
& =I_{1}+I_{2}, \\
I_{1} & =-\frac{1}{2 \imath} \int_{M, \frac{2}{2 \pi}} \psi_{0}(\rho s) \frac{z^{s} d s}{s \sin \pi s} \\
I_{2} & =-\frac{1}{2 \imath} \int_{M} Q(\rho s) \frac{z^{s} d s}{s \sin \pi s}
\end{array}\right\} .
$$

and

Let $M^{\prime}$ be the line $s=-1+a$. For all points $s$ within or upon the boundaries of the strip between $M$ and $M^{\prime}$, and outside a small circle excluding the origin, we have

$$
\begin{aligned}
& \left|Q(\rho s) \frac{z^{s}}{s \sin \pi s}\right| \\
& \quad<K \exp \left[|s|^{1 /(k-1)+e}\right] \exp [-|s| / K],
\end{aligned}
$$

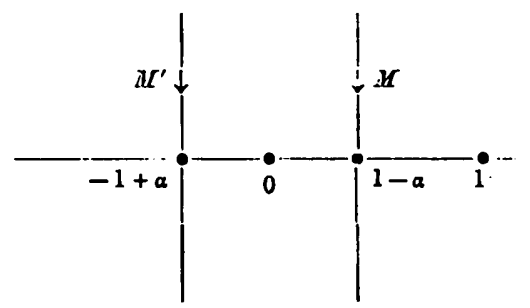

and therefore, since $\epsilon$ may be chosen so that $1 /(k-1)+\epsilon<1$,

$$
-\frac{1}{2 \imath}\left(\int_{M}-\int_{M}\right)\left(Q(\rho s) \frac{z^{s} d s}{s \sin \pi s}\right)
$$

exists, and, since the integrand is uniform, is equal to

$-2 \pi \iota\left[\right.$ the sum of the residues of $-\frac{1}{2 \iota} Q(\rho s) \frac{z^{s}}{s \sin \pi s}$

at the singularities between $M$ and $\left.M^{\prime}\right]$.

Now the only singularity within the strip is at $s=0$; also we have by I., $\S 5$,

$$
Q(\rho s)=-\frac{1}{2}-\rho\left[\xi(-1 / k)+\Sigma \rho_{\nu} \xi\left(-1 / k_{\nu}\right)\right] s+A s^{2}+\ldots .
$$

Then we have

$$
\begin{aligned}
-\frac{1}{2 \iota}\left(\int_{M}-\int_{M^{\prime}}\right) & \left(Q(\rho s) \frac{z^{s} d s}{s \sin \pi s}\right) \\
= & -\frac{1}{2} \log z-\rho\left[\zeta(-1 / k)+\Sigma \rho_{\nu} \xi\left(-1 / k_{\nu}\right)\right] .
\end{aligned}
$$

and

$$
I_{2}=-\frac{1}{2} \log z-\rho\left[\xi(-1 / k)+\Sigma \rho_{\nu} \xi\left(-1 / k_{\nu}\right)\right]+J \text {, }
$$

where

$$
J=-\frac{1}{2 \iota} \int_{M} Q(\rho s) \frac{z^{s} d s}{s \sin \pi s} .
$$


It may be shown, on the lines of $\$ 3$, that

$$
\lim _{i=1 \rightarrow \infty}\left|J z^{-1+a+e}\right|=0,
$$

and therefore that, for any value of $N_{1}$,

$$
\lim _{|z| \rightarrow \infty}\left|J(\log z)^{N_{1}}\right|=0 .
$$

We have now to consider $I_{1}$.

Let $1-\beta$ be a positive number less than $1-\alpha$. Let $M_{1}, M_{2}$ be the straight lines from $-1+\beta+\infty$, to $-1+\beta$ and $-1+\beta$ to $-1+\beta-\infty$, respectively, and let $T_{0}$ be the circle $|s|=1-\beta$, starting from $-1+\beta$ and described clockwise. Now upon and within the contours consisting of $M_{1}, T_{0}, M_{2}$, and $M$, we have

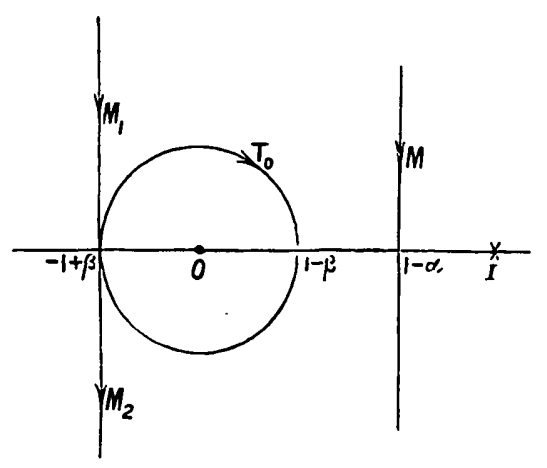

$$
\left|\psi_{0}(\rho s)\right|<K \exp \left[|s|^{e}\right],
$$

and

$$
\left|\frac{z^{s}}{s \sin \pi s}\right|<K \exp [-|s| / K] .
$$

It follows that the integral

exists.*

$$
\int_{M+\left(-M_{9}\right)+\left(-T_{0}\right)+\left(-M_{1}\right)} \psi_{0}(\rho s) \frac{z^{s} d s}{s \sin \pi s}
$$

Since the integrand has no singularities in the region between $M$ and. $M_{1}+T_{0}+M_{2}$, and since the contour of integration may be consiaered closed by two lines crossing from $M$ to $M_{2}$ and from $M_{1}$ to $M$, the intexrais along which lines tend to zero as the lines are taken more and more distant from the origin, the above integral must have the veiue zero. Hence

$$
\begin{aligned}
I_{1}= & -\frac{1}{2 \iota} \int_{M_{,}, \pi} \psi_{0}(\rho s) \frac{z^{s} d s}{s \sin \pi s} \\
=-\frac{1}{2 \iota} \int_{T_{0, \pi}} \psi_{0}(\rho s) \frac{z^{s} d s}{s \sin \pi s} & -\frac{1}{2 \iota} \int_{M_{1,3 \pi}} \psi_{0}(\rho s) \frac{z^{s} a s}{s \sin \pi s} \\
& -\frac{1}{2 \iota} \int_{M_{2,-\pi}} \psi_{0}(\rho s) \frac{z^{s} d z}{s \sin \pi s}
\end{aligned}
$$

* I use $-L$ to denote the contour $L$ described in the reverse airection. 
1908.] AsYmptotic EXPansions OF InTEgRaL functions OF Zero ORDER. 253

Now upon $M_{1}$ and $M_{2}$, if $s=-(1-\beta)+\tau \iota$,

$$
\begin{aligned}
& \left|\psi_{0}(\rho s) / s\right|<K \exp \left[|s|^{e}\right]<K \exp \left[|\tau|^{e}\right], \\
& \left|\frac{z^{s}}{\sin \pi s}\right|<\frac{K\left|r^{s} e^{s, \theta}\right|}{\exp [-\pi|\tau|] / K} \quad\left(z=r e^{\iota \theta},|\theta|<\pi-\delta\right) \\
& <K r^{-1+\beta} \exp [-\delta|\tau|],
\end{aligned}
$$

where all the $K$ 's are independent of $z$ as well as of $s$. Therefore

$$
\left|-\frac{1}{2 \iota} \int_{M_{1}} \psi_{0}(\rho s) \frac{2^{s} d s}{s \sin \pi s}\right|<K r^{-1+\beta} \int_{-\infty}^{\infty} e^{-\delta|\tau|+\kappa|\tau|^{\alpha}} d \tau<K r^{-1+\beta}
$$

and a similar inequality holds for the integral along $M_{2}$.

Hence

$$
I_{1}=-\frac{1}{2 \imath} \int_{T, \pi} \psi_{0}(\rho s) \frac{z^{s} d s}{s \sin \pi s}+J^{\prime}
$$

where

$$
\lim _{|:| \rightarrow \infty \rightarrow \infty}\left|J^{\prime} z^{-1+\beta}\right|<K
$$

and therefore

$$
\lim _{|z| \rightarrow \infty}\left|J^{\prime}(\log z)^{N_{1}}\right|=0
$$

5. From (7), (8), (8)', (9), and (9)' we now have

$$
\left.\begin{array}{c}
\log F(z)=-\frac{1}{2} \log z-\rho\left[\xi(-1 / k)+\Sigma \rho_{\nu} \xi\left(-1 / k_{\nu}\right)\right]+U(z)+J^{\prime \prime}, \\
\lim _{|=| \rightarrow \infty} J^{\prime \prime}(\log z)^{N_{1}} i=0 \\
U(z)=-\frac{1}{2 \iota} \int_{T_{t, \pi} \pi} \psi_{0}(\rho s) \frac{z^{s} d s}{s \sin \pi s}
\end{array}\right\}
$$
where

and

It remains, then, to consider the integral $U(z)$.

We shall prove the following preliminary results.

For all positive values of $\lambda$, when $j z \mid>1$,

$$
\int_{T_{0}}\left|s^{\lambda} \frac{z^{s} d s}{s \sin \pi s}\right|<K(\lambda / e)^{\lambda}|\log z|^{-\lambda+2},
$$

where $K$ is independent of both $z$ and $\lambda$.

For any value of $\kappa$, positive or negative,

$$
\begin{aligned}
& \int_{T_{1,}, \pi} s^{-\kappa} \frac{z^{s} d s}{s \sin \pi s} \\
& =-\frac{2 \iota \sin \kappa \pi}{\pi}(\log z)^{\kappa+1}\left[\Gamma(-\kappa-1)+\sum_{n=1}^{N_{1}} \Gamma(2 n-\kappa-1) A_{2 n}(\log z)^{-2 n}\right]+{ }_{\kappa} J_{N_{1},}
\end{aligned}
$$


where $A_{2 n}$ is the coefficient of $t^{2 n}$ in the expansion of $\pi t / \sin \pi t$, and where, for all values of $N_{1}$,

$$
\lim _{z \mid \rightarrow \infty}\left|{ }_{\kappa} J_{N_{1}}(\log z)^{-\kappa-1+2 N_{1}}\right|=0 .
$$

We suppose that $r=|z|>\exp [1 /(1-\beta)]$, so that $(1-\beta) \log r>1$. Let $s=x / \log z$, and let $\phi=\arg (\log z)$. Since $\log r>0$, we have $|\phi|<\frac{1}{2} \pi$. Let $T_{0}^{\prime}$ be the contour in the $x$-plane corresponding to $T_{0}$ in the $s$-plane, so that $T_{0}^{\prime}$ is a circle described clockwise, starting from and returning to the point $-(1-\beta) \log z$. Let $T_{1}$ be an $x$ contour, described clockwise, starting from and returning to $-(1-\beta) \log z$ and circumscribing the origin, which is such that at all points of $T_{1}$ a $x<\mathrm{a}$ constant $h$ and $|x|>$ a constant $h^{\prime}$, which lies between the parallels distant $\pi$ from the real axis, and whose length is less than $2 \pi|\log z|$. Let $T_{2}$ be the straight line from $-\infty-(1-\beta) \log z$ to $-(1-\beta) \log z$, parallel to the real axis, and let $T_{3}$ be $T_{2}$ described in the reverse direction.

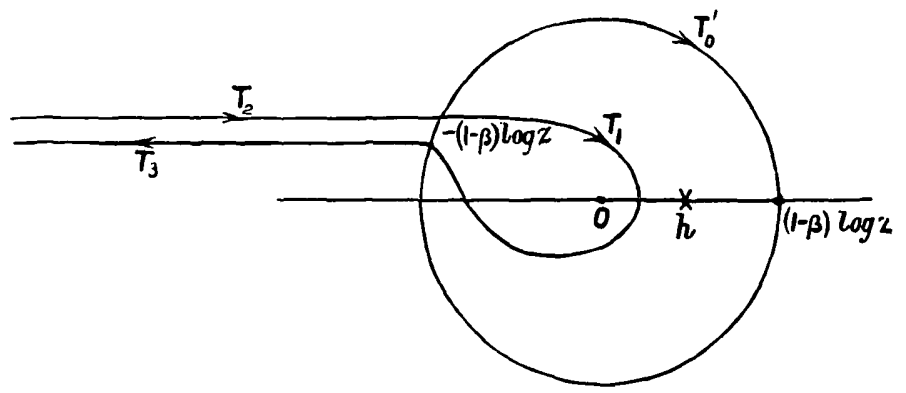

Consider the integral $\int_{T_{1}}\left|x^{\lambda} e^{x} d x\right|$. On $T_{1}$ we have

$$
\xi=\pi x<h, \text { and }|I x|<\pi .
$$

Therefore, for all points $x$ of $T_{1}$ we have

$$
\left|x^{\lambda} e^{x}\right|<K \xi^{\lambda} e^{\xi}
$$

where $K$ is independent of $\xi$ and $\lambda$. Now $\xi^{\lambda} e^{\xi}$ has its maximum modulus for $\hat{\xi} \leqslant h$, either when $\hat{\xi}=h$ or when $\hat{\xi}=-\lambda$. The value in the second case is $(\lambda / e)^{\lambda}$. Hence, on $T_{1}$, we have

$$
\left|x^{\lambda} e^{x}\right|<K(\lambda / e)^{\lambda}
$$

and therefore

$$
\int_{T_{1}}\left|x^{\lambda} e^{x} d x\right|<K(\lambda / e)^{\lambda}|\log z|,
$$

since the length of $T_{1}$ is less than $2 \pi|\log z|$. 
1908.] AsYMPTOTIC EXPANSIONS OF INTEGRAL FUNCTIONS OF ZERo ORDER. 255

Now for all points $x$ of $T_{1}$, we clearly have

$$
\left|\operatorname{cosec}\left(\frac{\pi x}{\log z}\right)\right|<K|\log z|
$$

since $|x|$ is greater than some constant $h^{\prime}$, and is not greater than $(1-\beta)|\log z|$. We therefore have

$$
\int_{T_{1}}\left|x^{\lambda-1} e^{x} d x \operatorname{cosec}\left(\frac{\pi x}{\log z}\right)\right|<K|\log z|^{2}\left(\frac{\lambda}{e}\right)^{\lambda}
$$

and therefore, putting $x=s \log z$,

$$
\int_{T_{1}}\left|s^{\lambda} \frac{z^{s} d s}{s \sin \pi s}\right|<K|\log z|^{-\lambda+2}\left(\frac{\lambda}{e}\right)^{\lambda}
$$

the first of our results.

Turning to the second result, we shall show that

$$
\int_{T_{1}, \pi+\phi} x^{-\kappa-2+2 n} e^{x} d x=-2 \iota \sin \kappa \pi \Gamma(2 n-\kappa-1)+J(n, \kappa),
$$

where

$$
\lim _{|z| \rightarrow \infty}\left|J(n, \kappa)(\log z)^{p}\right|=0,
$$

for all values of $p$.

The contour $T_{2}+T_{1}+T_{3}$ starts from and returns to $-\infty-(1-\beta) \log z$, and circumscribes the origin. Hence, by a well known result in the theory of the gamma-function,

$$
\begin{aligned}
\int_{T_{9}+T_{1}+T_{3}, \pi} x^{-\kappa-2+2 n} e^{x} d x & =-2 \iota \sin (-\kappa-1+2 n) \pi \cdot \Gamma(2 n-\kappa-1) \\
& =-2 \iota \sin \kappa \pi \cdot \Gamma(2 n-\kappa-1) .
\end{aligned}
$$

Now, if $x$ be any point of $T_{2}$ or $T_{3}, x=-\dot{\xi}-(1-\beta) \log z$, where $\xi \geqslant 0$, and hence

$$
\begin{aligned}
\left|x^{-\kappa-2+2 n} e^{x}\right| & <|x|^{2 n} e^{-(1-\beta) \log _{0} r-\xi} \quad[\text { since }|x| \geqslant(1-\beta) \log r>1] \\
& <r^{-(1-\beta)}|(1-\beta) \log z+\xi|^{2 n} e^{-\xi}
\end{aligned}
$$

whence it easily follows that

$$
\lim _{|z| \rightarrow \infty}\left|(\log z)^{p}\left\{\int_{T_{2}}+\int_{T_{3}}\right\} x^{-\kappa-2} e^{x} d x\right|=0
$$

From (5) $)_{1}$ and $(5)_{2}$, we obtain (5). 
When $|t|<1$, we have

where

$$
\begin{gathered}
\frac{\pi t}{\sin \pi t}=1+\sum_{n=1}^{\infty} A_{2 n} t^{2 n}, \\
A_{2 n}=2 \frac{\left(2^{2 n-1}-1\right) \pi^{2 n} B_{2 n}}{2 n !} .
\end{gathered}
$$

The radius of convergence of the series is 1 , and we have

$$
\left|A_{2 n}\right|<K\left[(1-\beta)^{k}\right]^{-2 n} ;
$$

and therefore, when $|t| \leqslant 1-\beta$,

$$
\left|\sum_{n=N_{1}+1}^{\infty} A_{2 n} t^{2 n}\right| \leqslant K \sum_{N_{1}+1}^{\infty}(1-\beta)^{-n}|t|^{2 n}<K|t|^{2 N_{1}+2},
$$

where $K$ is independent of $t$. Now, for all points $x$ of $T_{1}$, we have

therefore, by (6),

$$
|x / \log z| \leqslant 1-\beta
$$$$
\left(\frac{\pi x}{\log z}\right) \operatorname{cosec}\left(\frac{\pi x}{\log z}\right)=1+\sum_{n=1}^{N_{1}} A_{2 n}(\log z)^{-2 n} x^{3 n}+A_{2 N_{1}}(z)(\log z)^{-2 N_{1}-2} x^{2 N_{1}+2},
$$

where $\left|A_{2 N_{1}}(z)\right|<$ a $K$ independent of $x$ and $z$. Hence

$$
\begin{aligned}
\int_{T_{1}} e^{x} x^{-\kappa-2}\left(\frac{\pi x}{\log z}\right) \operatorname{cosec}\left(\frac{\pi x}{\log z}\right) d x & \\
=\int_{T_{1}} e^{x} x^{-\kappa-2} d x & +\sum_{n=1}^{\dot{r}_{1}} A_{2 n}(\log z)^{-2 n} \int_{T_{1}} e^{x} x^{-\kappa-2+2 n} d x \\
& \quad+\int_{T_{1}} A_{2 N_{1}}(z)(\log z)^{-2 N_{1}-2} x^{2 N_{1}-\kappa} e^{x} d x ;
\end{aligned}
$$

and therefore, by (5),

$$
\begin{aligned}
=-2 \iota & \sin \kappa \pi \Gamma(-\kappa-1)+J(0, \kappa) \\
& +\sum_{n=1}^{N_{1}}\left[-2 \iota \sin \kappa \pi \Gamma(2 n-\kappa-1) A_{2 n}(\log z)^{-2 n}+J(n, \kappa)\right]+J^{\prime}\left(N_{1}, \kappa\right),(7)
\end{aligned}
$$

where $J^{\prime}\left(N_{1}, \kappa\right)$ is the integral last written. Now

$$
\lim _{l: \rightarrow \infty}\left|(\log z)^{2 N_{1}} J^{\prime}\left(N_{1}, \kappa\right)\right| \leqslant \lim _{z: \rightarrow \rightarrow \infty} K|\log z|^{-2} \int_{T_{1}}\left|x^{2 N_{1}-\kappa} e^{x} d x\right|=0,
$$

by means of (4), provided that we suppose $2 N_{1}-\kappa>0$. Hence, since

$$
\lim _{|z| \rightarrow \infty}\left|(\log z)^{p} J(n, \kappa)\right|=0,
$$


for all values of $p$, we have

$$
\lim _{|z| \rightarrow \infty}\left|(\log z)^{2 N_{1}}\left[J(0, \kappa)+\sum_{n=1}^{N_{1}} J(n, \kappa)+J^{\prime}\left(N_{1}, \kappa\right)\right]\right|=0 .
$$

Now if in the integral $\int_{T_{0, \pi}} s^{-\kappa} \frac{z^{s} d s}{s \sin \pi s}$ we make the transformation $s=x / \log z$, we obtain the integral on the left-hand side of (7), multiplied by $\pi(\log z)^{-\kappa-1}$. Then, from (7) and (8), we obtain at once the desired result (3).

Now let us consider $U(z)$. We have

$$
\begin{aligned}
U(z) & =-\frac{1}{2 \imath} \int_{T_{0}} \frac{\psi_{0}(\rho s) z^{s}}{s \sin \pi s} d s \\
& =-\frac{1}{2 \iota} \int_{T_{0}} \frac{(\rho s)^{-k} z^{s} d s}{s \sin \pi s}\left[\sum_{n=0}^{\infty} c_{n}(\rho s)^{\theta_{n}}\right],
\end{aligned}
$$

where we have

$$
c_{0}=\Gamma(k+1), \quad \theta_{0}=0 \text {. }
$$

Since the infinite series is uniformly convergent,

$$
\begin{aligned}
U(z) & =-\sum_{n=0}^{\infty} \frac{c_{n} \rho^{\theta_{n}-k}}{2 \iota} \int_{T_{n}} \frac{s^{\theta_{n}-k} z^{s}}{s \sin \pi s} d s \\
& =-\sum_{n=0}^{N_{2}} \frac{c_{n} \rho^{\theta_{n}-k}}{2 \iota} \int_{T_{0}} \frac{s^{\theta_{n}-k} z^{s}}{s \sin \pi s} d s+J\left(N_{2}\right),
\end{aligned}
$$

where $N_{2}$ is any integer such that $\theta_{N_{2}}-k>e$, and where

$$
J\left(N_{2}\right)=-\sum_{n=\Lambda s+1}^{\infty} \frac{c_{n} \rho^{\theta_{n}-k}}{2 \iota} \int_{T_{0}} \frac{s^{\theta_{n}-k} z^{s}}{s \sin \pi s} d s .
$$

Now the modulus of the coefficient of $c_{n} \rho^{\theta_{n}-k}$ in the last series is les: than $K(\log z)^{2+l i-\theta_{n}}\left(\frac{\theta_{n}-k}{e}\right)^{\theta_{n}-k}$, by (2). Hence

$$
\begin{aligned}
\left|J\left(N_{2}\right)\right|<K|\log z|^{2+k} \sum_{n=N_{2}+1}^{\infty}\left|c_{n}\right| \rho^{\theta_{n}-k}|\log z|^{-\theta_{n}}\left(\theta_{n} / e\right)^{\theta_{n}} & \quad\left(\text { since } \overline{\theta_{n}-k} / e>1\right) \\
& <K|\log z|^{2+k} \sum_{n=N_{2}+1}^{\infty}\left(K / \theta_{n}\right)^{\theta_{n}}|\log z|^{-\theta_{n}}\left(\theta_{n} / e\right)^{\theta_{n}},
\end{aligned}
$$

skr. 2. voL. 7 . No. 1021. 
by the inequality (iv.) of $\mathrm{I} ., \S 5$,

$$
\begin{aligned}
& <K|\log z|^{2+k} \sum_{N_{2}+1}^{\infty}\left[\frac{K}{|\log z|}\right]^{\theta_{n}} \\
& <K|\log z|^{2+k-\theta_{N_{2}+1}} \sum_{N_{2}+1}^{\infty}\left[\frac{K}{|\log z|}\right]^{-\theta_{11}+\theta_{N_{2}+1}} \\
& \left.<K|\log z|^{2+k-\theta_{N_{2}+1}} \sum_{N_{2}=1}^{\infty} e^{-\theta_{n}+\theta_{N_{2}+1}} \quad \text { (provided } K<e|\log z|\right) \\
& <K|\log z|^{2+k-\theta_{N_{2}+1}}
\end{aligned}
$$

$\sum e^{-\theta_{n}}$ being convergent, since $\theta_{n}>K^{-1} n^{1:(\mu+1)}$.

Now if we replace each term $\int_{T_{0}} \frac{s^{\theta_{n}-k} z^{s}}{s \sin \pi s} d s$ on the right-hand side of (9) by its equivalent given by (3), in which $\kappa$ is to be replaced by $k-\theta_{n}$. we obtain

$$
\begin{aligned}
U(z)=\sum_{n=0}^{N_{2}} \rho c_{n} & \frac{\sin \left(k-\theta_{n}\right) \pi}{\pi}\left(\frac{\log z}{\rho}\right)^{k-\theta_{n}+1} \\
& \times\left[\Gamma\left(-l i+\theta_{n}-1\right)+\sum_{m=1}^{N_{1}} \Gamma\left(2 m-k+\theta_{n}-1\right) \cdot A_{2 m}(\log z)^{-2 m}\right] \\
& +\sum_{n=0}^{N} k_{i=\theta_{n}} J_{N_{\mathrm{L}}}+J\left(N_{2}\right),
\end{aligned}
$$

where we shall have

$$
\lim _{\mid z i \rightarrow \infty}\left|(\log z)^{p}\left[\sum_{n=0}^{N_{2}} k-\theta_{n} J_{N_{1}}+J\left(N_{2}\right)\right]\right|=0
$$

for any given $p$, provided $N_{1}$ and $N_{2}$ are chosen sufficiently large. Moreover, all terms in the finite sum in (11) which are of degree in $\log z$ less than $-p$ may be absorbed into the remainder term, for they clearly satisfy an inequality similar to that last written. If we now substitute this asymptotic expansion for $U(z)$ in (1), we obtain the expansion for $\log F(z)$ stated at the beginning of the last article, and it is clear that if we take any number of terms of the expansion arranged in order of increasing indices of $(\log z)^{-1}$, if the last term be of order $(\log z)^{-1}$, we shall have

$$
\lim _{|z| \rightarrow \infty}\left|(\log z)^{p} R\right|=0,
$$

where $R$ is the remainder. 


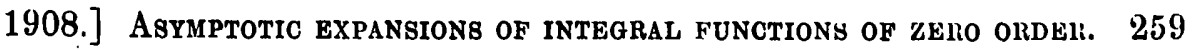

6. The expansion found for $\log F(z)$ has been proved for the case when $k>2$, and when $\rho, \rho_{1}, \rho_{2}, \ldots$ are real. It may be extended to the case when $\rho$ is complex, and $\rho_{1}, \rho_{2}, \ldots$ are real, the variable $z$ being excluded from a spiral strip, as in $\S \approx$, instead of an angle of the plane; the modified argument follows the lines of $\$ 3$. If, however, we suppose $\rho_{1}, \rho_{2}, \ldots$ complex, considerable difficulties appear to arise. And when we suppose $k<2$, our line of proof breaks down, because we cannot then prove $\mid S(\rho s)\}<K \exp [\epsilon|s|]$ in the region $2 s>-l$. It is almost certain, indeed, that this inequality does not then hold. The expansion, as a matter of fact, is none the less valid.*

7. In the case of the function

$$
F(z)=\prod_{n=1}^{\infty}\left\{1+z / \exp \left[\rho\left(n^{1 / k}+\rho_{1} n^{1 / k_{1}}\right)\right]\right\},
$$

we have

$$
\rho_{2}=\rho_{3}=\ldots=0,
$$

and $(\mathrm{I} ., \S 6)$

$$
\sum_{n=0}^{\infty} c_{n} s^{\theta_{n}}=\Gamma(k+1)+k \sum_{n=1}^{\infty} \frac{\left(-\rho_{1}\right)^{n} s^{n\left(1-k ; k_{2}\right)}}{\Gamma(n+1)} \Gamma\left(n k / k_{1}+k\right) .
$$

Then we have the expansion

$$
\begin{aligned}
& \log F(z)=-\rho\left[\xi(-1 / k)+\rho_{1} \xi\left(-1 / k_{1}\right)\right]-\frac{1}{2} \log z \\
& \quad+\frac{k \rho}{\pi}\left(\frac{\log z}{\rho}\right)^{k+1} \sum_{n=0} \sin \left(k+n k / k_{1}\right) \pi \cdot \Gamma\left(n k / k_{1}+k\right) \frac{\left(-\rho_{1}\right)^{n}}{n !}\left(\frac{\log z}{\rho}\right)^{-n\left(1-k k_{1}\right)} \\
& \times\left[\Gamma\left\{n\left(1-k / k_{1}\right)-k-1\right\}\right. \\
& \quad+2 \sum_{m=1}^{\sum} \Gamma\left\{2 m+n(1-k / k,-k-1\} \frac{\left(2^{2 m-1}-1\right) \pi^{2 m}}{2 m !} B_{2 m}(\log z)^{-2 m}\right] .
\end{aligned}
$$

In the case $\rho_{1}=0$, in which

$$
F(z)=\prod_{n=1}^{\infty}\left[1+z / \exp \left(\rho n^{1 / k}\right)\right],
$$

* See the Abstract, p. $21 \mathrm{~S}$. 
the expansion may be written

$\log F(z)=-\rho \xi(-1 / k)-\frac{1}{2} \log z$

$$
+\frac{\rho}{k+1}\left(\frac{\log z}{\rho}\right)^{k+1}\left[1+2 \sum_{n=1}^{k+1} C_{2 n}\left(2^{2 n-1}-1\right) \pi^{2 n} B_{2 n}(\log z)^{-2 n}\right] .
$$

8. It is not possible to apply the foregoing methods to determine an asymptotic expansion for the functions $F(z)$ whose $n$-th zero is of less order than $\exp \left(n^{k}\right)$ for all values of $k$. Consider, for example, the function

$$
F(z)=\prod_{n=2}^{\infty}\left[1+z /\left\{\exp (\log n)^{1+h}\right\}\right]
$$

In this case $S(s)$ contains the terms

$$
\sum_{1}^{\infty} A_{n} s^{-n(1+l)} \text {. }
$$

The term $s^{-i n(1+k)}$ gives rise to terms in $\log F(z)$ of the type

$$
\left[\sum_{n} C_{m}(\log z)^{-2 m}\right](\log z)^{n(1+1) \cdot+1}
$$

the series being an asymptotic one. An infinite series of such series cannot be combined into an asymptotic expansion. Moreover, if

$$
\lim _{n \rightarrow \infty}\left|a_{n} e^{-n^{c}}\right|=0
$$

for all values of $\epsilon$, however small, it may be shown from arithmetic considerations that the maximum positive value of $\log \left|\Pi\left(1+2 a_{n}\right)\right|$ is of higher order in $z$ than $(\log z)^{\prime \prime}$, for all values of $p$, so that if $\log F(z)$ possesses an expansion in terms of $\log z$, it must involve an integral function of $\log z$.

[We obtain, in fact, by the general theory referred to in the Abstract, for the function

$$
\varlimsup_{n=:}^{\varkappa}\left[1+z / \exp \left\{\rho(\log n)^{1+k}\right\}\right] \text {, }
$$


1908.] Asymptotic EXPANgIONS OF INTEGRAL FUNCTIONS OF ZERO ORDER. 261

$$
\begin{aligned}
& \log F(z)=\exp \left\{\left(\frac{\log z}{\rho}\right)^{1 /(1+k)} ;\right. \\
& \times\left[\rho(1+k)\left\{\left(\frac{\log z}{\rho}\right)^{k /(1+k)}-k\left(\frac{\log z}{\rho}\right)^{(k-1)(k+1)}+k(k-1)\left(\frac{\log z}{\rho}\right)^{(k-2)(k+1)}+\ldots\right\}\right. \\
& \left.+\frac{\pi^{2}}{6 \rho(1+k)}\left(\frac{\log z}{\rho}\right)^{-k(1+k)}+\text { lower terms }\right] .
\end{aligned}
$$

The terms within square brackets may be found as far as any numerically assigned index of $1 / \log z$, and give an asymptotic though probably divergent expansion. But this expansion is of a different character from those we have been considering, as it involves the factor $\left.\exp \left\{(\log z / \rho)^{1 .(1+k)}\right\}\right]$.*

It may be noted, in conclusion, that it is possible to find asymptotic expansions for certain forms of $F(z)$ whose zeros are repeated, and whose $n$-th zeros are of the order $\exp \left(n^{1}\right), k>1$. The function

$$
\prod_{n=1}^{\infty}\left[1+\frac{z}{\exp \left(n^{1 \cdot k}\right)}\right]^{n^{n}}
$$

is an example.t Its Dirichlet series defines the function

$$
S(s)=k \Gamma(k \lambda+k) s^{-k \lambda-k}+\sum_{n=0}^{\infty} \frac{(-s)^{n}}{\Gamma(n+1)} \xi(-\lambda-n / k),
$$

as is seen by replacing $\lambda s$ by $\lambda$ in the result of I., $\$ 1$. We may now proceed as in II., $\$ 2$, and if $k \lambda+k$ is an integer and $k>2$, we obtain an asymptotic expansion for $\log F(z)$ analogous to that of that article.

[I take this opportunity of correcting an error in a proof in my paper "On the Asymptotic Approximation to Integral Functions of Zero Order," which appeared in these Proceedings, Ser. 2, Vol. 5, pp. 361-410.

From p. 380 , 1. 5, to p. $381,1.3$, should be replaced by the following argument.

We have

$$
\frac{\lambda}{2 \eta} \frac{s-1}{n}<\frac{\lambda}{2 \eta} \frac{\eta n}{n}<\frac{1}{2} \lambda
$$

Hence

$$
1-\exp \left(-\frac{\lambda}{2 \eta} \frac{s-1}{n}\right)>\frac{1}{K}\left(\frac{\lambda}{2 \eta} \frac{s-1}{n}\right) \text {, }
$$

- Added February 8th, 1909.

t The rank of the zero $-\exp \left(\rho n^{i k}\right)$ is not $n$ but $1+\left(1^{\lambda}+2^{\lambda}+\ldots+\overline{n-1^{\lambda}}\right)$. The $n$-th zero is, however, of the order $\exp \left[n^{\prime}(k \lambda+1)\right]$. 
262 ASYMIPTOTIC EXPANSIONS OF INTEGRAL FUNCTIONS OF ZERO ORDER. [Nov. 12, where $K$ is a constant (depending on $\lambda$ ) which is independent of $n$, for $\left(1-e^{-x}\right) / x$ lies between finite limits when $x<\frac{1}{2} \lambda$. Hence, from (4),

$$
1 /\left[1-\left(\frac{n+1}{n+s}\right)^{\lambda^{\prime} \eta \eta}\right]<K /\left(\frac{\lambda}{2 \eta} \frac{s-1}{n}\right)<\frac{K^{\prime} \eta n}{s-1} .
$$

Then, by a slight modification of 1.14 , p. 380 , to 1.3 , p. 381 , we obtain

$$
\left|\sigma_{1}\right|<K^{\prime \prime} \cdot m+K^{\prime \prime \prime} \text {. }
$$

This result then leads as in the text to (6), provided $K_{1}$ is suitably chosen. A similar modification must be made in the case of $\sigma_{8}$, p. 383, 1l. 1-8.] 